\title{
Signs of outflow feedback from a nearby young stellar object on the protostellar envelope around HL Tauri
}

\author{
Hsi-Wei Yen ${ }^{1,2}$, Shigehisa Takakuwa ${ }^{3,2}$, Pin-Gao Gu${ }^{2}$, Naomi Hirano ${ }^{2}$, Chin-Fei Lee ${ }^{2}$, \\ Hauyu Baobab Liu ${ }^{1,2}$, Sheng-Yuan $\mathrm{Liu}^{2}$, and Chun-Ju Wu${ }^{4,2}$ \\ ${ }^{1}$ European Southern Observatory (ESO), Karl-Schwarzschild-Str. 2, 85748 Garching, Germany \\ 2 Academia Sinica Institute of Astronomy and Astrophysics, 11F of Astro-Math Bldg, 1, Sec. 4, Roosevelt Rd, Taipei 10617, Taiwan \\ e-mail: hwyen@asiaa.sinica.edu.tw \\ ${ }^{3}$ Department of Physics and Astronomy, Graduate School of Science and Engineering, Kagoshima University, 1-21-35 Korimoto, \\ Kagoshima, Kagoshima 890-0065, Japan \\ ${ }^{4}$ Department of Physics, National Taiwan University, No. 1, Sec. 4, Roosevelt Road, Taipei 106, Taiwan
}

Received 8 September 2018 / Accepted 10 January 2019

\begin{abstract}
Aims. HL Tau is a Class I-II protostar embedded in an infalling and rotating envelope and possibly associated with a planet forming disk, and it is co-located in a $0.1 \mathrm{pc}$ molecular cloud with two nearby young stellar objects with projected distance of $\sim 20^{\prime \prime}-30^{\prime \prime}$ (2800-4200 au) to HL Tau. Our observations with the Atacama Large Millimeter/Submillimeter Array (ALMA) revealed two arc-like structures on a 1000 au scale connected to the disk, and their kinematics could not be explained with any conventional model of infalling and rotational motions. In this work, we investigate the nature of these arc-like structures connected to the HL Tau disk.

Methods. We carried out new observations in the ${ }^{13} \mathrm{CO}$ and $\mathrm{C}^{18} \mathrm{O}(3-2 ; 2-1)$ lines with the James Clerk Maxwell Telescope and the IRAM 30m telescope, and obtained the data with the 7-m array of the Atacama Compact Array (ACA). With the single-dish, ACA, and ALMA data, we analyzed the gas motions on both $0.1 \mathrm{pc}$ and 1000 au scales in the HL Tau region. We constructed new kinematical models of an infalling and rotating envelope with the consideration of relative motion between HL Tau and the envelope.

Results. By including the relative motion between HL Tau and its protostellar envelope, our kinematical model can explain the observed velocity features in the arc-like structures. The morphologies of the arc-like structures can also be explained with an asymmetric initial density distribution in our model envelope. In addition, our single-dish results support the scenario that HL Tau is located at the edge of a largescale $(0.1 \mathrm{pc})$ expanding shell driven by the wind or outflow from XZ Tau, as suggested in the literature. The estimated expanding velocity of the shell is comparable to the relative velocity between HL Tau and its envelope in our kinematical model. These results hint that the largescale expanding motion likely impacts the protostellar envelope around HL Tau and affects its gas kinematics. We found that the mass infalling rate from the envelope onto the HL Tau disk can be decreased by a factor of two due to this impact by the largescale expanding shell.
\end{abstract}

Key words. protoplanetary disks - ISM: kinematics and dynamics - stars: protostars - stars: formation - circumstellar matter

\section{Introduction}

HL Tau is a Class I-II protostar (Men'shchikov et al. 1999; Motte \& André 2001; Furlan et al. 2008) located in the Taurus star-forming region at a distance of 140 pc (Kenyon et al. 1994; Loinard 2013; Galli et al. 2018). HL Tau is surrounded by a protostellar envelope with a size of 2000 au (Hayashi et al. 1993). This envelope is embedded in a molecular cloud with a length of $0.05 \mathrm{pc}$ elongated along the northwest-to-southeast direction (Welch et al. 2000). The protostellar envelope around HL Tau exhibits signs of infalling and rotational motions on a $1000 \mathrm{au}$ scale as observed in the ${ }^{13} \mathrm{CO}(1-0)$ emission at an angular resolution of 5" (Hayashi et al. 1993). Nevertheless, the overall velocity structures in the protostellar envelope are complex (Cabrit et al. 1996; Welch et al. 2000; Wu et al. 2018). Bipolar molecular outflows and high-velocity $\left(60-160 \mathrm{~km} \mathrm{~s}^{-1}\right)$ optical and infrared jets associated with HL Tau are observed (Monin et al. 1996; Takami et al. 2007; Hayashi \& Pyo 2009; Anglada et al. 2007; Movsessian et al. 2012; Lumbreras \& Zapata 2014; ALMA Partnership 2015; Klaassen et al. 2016). The presence of the infalling envelope and the outflow activities suggests that
HL Tau is still in the main accretion phase and is less evolved compared to other Class II protostars.

Observations in the (sub-)millimeter continuum with the Atacama Large Millimeter/Submillimeter Array (ALMA) revealed a series of rings and gaps in the circumstellar disk around HL Tau (ALMA Partnership 2015; Akiyama et al. 2016). The presence of such rings and gaps can be a signpost of gas giant planets embedded in the disk (e.g., Dong et al. 2015, 2016; Kanagawa et al. 2015, 2016; Dong \& Fung 2017). Imaging observations in the $L^{\prime}$ band with the Large Binocular Telescope Interferometer found no point sources in the disk, and the upper limit on the mass of the putative planets is estimated to be 10-15 $M_{\text {jup }}$ (Testi et al. 2015). Nevertheless, this upper limit is more than ten times higher than the estimated required mass for a planet to carve the observed gaps in the disk around HL Tau (Kanagawa et al. 2015; Akiyama et al. 2016; Jin et al. 2016; Yen et al. 2016). These observational results make HL Tau one of the youngest candidates for ongoing planet formation.

When there is material infalling onto a protoplanetary disk from its surrounding envelope, the disk mass increases, which may cause the disk to become gravitationally unstable 
Table 1. Summary of images.

\begin{tabular}{cccccc}
\hline \hline Telescopes & Line & Field of view & Velocity resolution & Angular resolution & Noise per channel \\
\hline JCMT & ${ }^{13} \mathrm{CO}(3-2)$ & $2^{\prime} \times 2^{\prime}$ & $0.06 \mathrm{~km} \mathrm{~s}^{-1}$ & $15^{\prime \prime} 3$ & $0.44 \mathrm{~K}$ \\
JCMT & $\mathrm{C}^{18} \mathrm{O}(3-2)$ & $2^{\prime} \times 2^{\prime}$ & $0.06 \mathrm{~km} \mathrm{~s}^{-1}$ & $15^{\prime \prime} 2$ & $0.41 \mathrm{~K}$ \\
IRAM 30m & ${ }^{13} \mathrm{CO}(2-1)$ & $2^{\prime} \times 2^{\prime}$ & $0.07 \mathrm{~km} \mathrm{~s}^{-1}$ & $11^{\prime \prime} 8$ & $0.24 \mathrm{~K}$ \\
IRAM 30m & $\mathrm{C}^{18} \mathrm{O}(2-1)$ & $2^{\prime} \times 2^{\prime}$ & $0.07 \mathrm{~km} \mathrm{~s}^{-1}$ & $11^{\prime \prime} 8$ & $0.26 \mathrm{~K}$ \\
30m+ACA+ALMA & ${ }^{13} \mathrm{CO}(2-1)$ & $40^{\prime \prime} \times 40^{\prime \prime}$ & $0.2 \mathrm{~km} \mathrm{~s}^{-1}$ & $1^{\prime \prime} \times 0^{\prime} \cdot 9\left(151^{\circ}\right)$ & $7.7 \mathrm{mJy} \mathrm{Beam}^{-1}$ \\
$30 \mathrm{~m}+$ ACA+ALMA & $\mathrm{C}^{18} \mathrm{O}(2-1)$ & $40^{\prime \prime} \times 40^{\prime \prime}$ & $0.34 \mathrm{~km} \mathrm{~s}^{-1}$ & $1^{\prime \prime} \times 0^{\prime} 9\left(160^{\circ}\right)$ & $5.5 \mathrm{mJy} \mathrm{Beam}^{-1}$ \\
\hline
\end{tabular}

(Machida et al. 2010; Vorobyov 2010). This may lead to the formation of progenitors of planets or mass ejection from the disk (Vorobyov 2011, 2016; Zhu et al. 2012). Infalling material onto protoplanetary disks can also produce accretion shock and affect the chemistry in the disks (Visser et al. 2009, 2011). In order to study the gas kinematics of the protostellar envelope around HL Tau and its influence on the HL Tau disk, we conducted ALMA observations in the ${ }^{13} \mathrm{CO}(2-1)$ and $\mathrm{C}^{18} \mathrm{O}(2-1)$ lines at an angular resolution of 0.' 8 (Yen et al. 2017).

Our previous ALMA observations revealed two arc-like structures with sizes of 1000 and 2000 au and masses of $3 \times 10^{-3} M_{\odot}$ connected to the HL Tau disk in the protostellar envelope. One is blueshifted and stretches from the northeastern part of the disk toward the northwestern direction, and the other is redshifted and stretches from the southwestern part of the disk toward the southeastern direction. The kinematics of the arc-like structures could not be explained by conventional models of an infalling and rotating envelope. In addition, the observed velocity in the blueshifted arc-like structure is higher than the expected free-fall velocity in HL Tau. The origins of the arc-like structures are unclear. Two possibilities are suggested in Yen et al. (2017), infalling flows under the external compression or outflowing gas caused by gravitational instabilities in the disk.

To investigate the origins of the arc-like structures connected to the HL Tau disk, we conducted observations in the ${ }^{13} \mathrm{CO}(3-2)$ and $\mathrm{C}^{18} \mathrm{O}(3-2)$ lines with the James Clerk Maxwell Telescope (JCMT) and in the ${ }^{13} \mathrm{CO}(2-1)$ and $\mathrm{C}^{18} \mathrm{O}(2-1)$ lines with the IRAM $30 \mathrm{~m}$ telescope and mapped the kinematics of the largescale molecular cloud associated with HL Tau. In addition, we have obtained the data of the ${ }^{13} \mathrm{CO}(2-1)$ and $\mathrm{C}^{18} \mathrm{O}(2-1)$ lines with the 7-m array of the Atacama Compact Array (ACA), which is a part of our ALMA project. With the IRAM $30 \mathrm{~m}$, ACA, and ALMA data, we generated combined images. In this paper, we present the single-dish and combined images, and we discuss the physical conditions and kinematics on $0.1 \mathrm{pc}$ and 1000 au scales. To explain the observed velocity features in the arc-like structures, we construced a new kinematical model for the protostellar envelope around HL Tau with the consideration of relative motion between HL Tau and the envelope. We discuss the possible origin of this relative motion and its effects on the dynamics and evolution of the protostellar envelope around HL Tau.

\section{Observations}

The fields of view, the velocity and angular resolutions, and the noise levels of all the observations are summarized in Table 1. In this paper, the intensity of the single-dish data is presented in units of main beam brightness temperature.

\subsection{JCMT observations}

Our observations in the ${ }^{13} \mathrm{CO}(3-2 ; 330.587960 \mathrm{GHz})$ and $\mathrm{C}^{18} \mathrm{O}(3-2 ; 329.330545 \mathrm{GHz})$ lines toward HL Tau with JCMT were conducted in 2017 on January 14, 17, 29, and 30, February 5, and August 1. The observations were conducted in the grade 3 weather with $225 \mathrm{GHz}$ opacity ranging from 0.08 to 0.12 . The on-source observing time on each day ranges from 0.3 to $1.4 \mathrm{~h}$, and the total on-source time is $4.4 \mathrm{~h}$. The Heterodyne Array Receiver Program (HARP) was adopted. HARP is an array receiver with $4 \times 4$ detectors, which are named as receptor H00, H01 to H15. The main beam efficiency of HARP is 0.64 . The receptors, $\mathrm{H} 13$ and $\mathrm{H} 14$, were not operational. The data taken with the receptors $\mathrm{H} 04$ on all the observing days, H08 on January 14, and H03 on February 5 were not usable, and they were excluded in our data reduction. The observations were conducted with the jiggle-position switch mode, which covers an area of $2^{\prime} \times 2^{\prime}$ centered at the position of HL Tau. The spectrometer ACSIS was adopted as the backend and was configured to have two spectral windows in the $250 \mathrm{MHz}$ mode to observe the ${ }^{13} \mathrm{CO}(3-2)$ and $\mathrm{C}^{18} \mathrm{O}(3-2)$ lines simultaneously. In each spectral window, the usable bandwidth is $220 \mathrm{MHz}$, and the channel number and width are 4096 and $61 \mathrm{kHz}$, respectively. That corresponds to a velocity resolution of $0.06 \mathrm{~km} \mathrm{~s}^{-1}$ at the frequencies of the ${ }^{13} \mathrm{CO}(3-2)$ and $\mathrm{C}^{18} \mathrm{O}(3-2)$ lines. The data were reduced with Starlink (Currie et al. 2014) and the ORAC-DR pipeline (Jenness et al. 2015), and the recipe of "reduce_science_narrowline" was adopted. This recipe is designed for data with one or more narrow $\left(<8 \mathrm{~km} \mathrm{~s}^{-1}\right)$ emission lines in the observed frequency band. In particular, it smooths data by $5 \times 5$ pixels $\times 10$ channels to find regions for fitting and subtracting baselines ${ }^{1}$. The achieved noise level in main beam temperature is $0.4 \mathrm{~K}$ at a velocity resolution of $0.06 \mathrm{~km} \mathrm{~s}^{-1}$.

\subsection{IRAM $30 \mathrm{~m}$ observations}

Our observations toward HL Tau with the IRAM 30m telescope were conducted on March 28 and 29 in 2017. During the observations, the $225 \mathrm{GHz}$ opacity ranged from 0.08 to 0.15 . The total on-source time was $4.7 \mathrm{~h}$. The observations were conducted with the EMIR receiver. On-the-fly mapping was performed to cover an area of $2^{\prime} \times 2^{\prime}$ centered at the position of HL Tau. Dual bands, $\mathrm{E} 90$ and E230, at 3 and $1.3 \mathrm{~mm}$ with dual polarizations were observed simultaneously. The spectrometer FTS was adopted as the backend and was configured in the narrow mode, resulting in a bandwidth of $1.8 \mathrm{GHz}$ and a spectral resolution of $50 \mathrm{kHz}$ for each frequency band and each polarization. The observed frequency ranges covered several molecular lines, including $\mathrm{HCO}^{+}$ $(1-0), \mathrm{H}^{13} \mathrm{CO}^{+}(1-0), \mathrm{HCN}(1-0)$, and $\mathrm{SiO}(2-1)$ in the E90

1 The detailed process is described at http://www. starlink.ac. $\mathrm{uk} / \operatorname{devdocs} / \operatorname{sun} 260 \cdot \mathrm{htx} / \operatorname{sun} 260 \cdot \mathrm{html}$ 
band and ${ }^{13} \mathrm{CO}(2-1), \mathrm{C}^{18} \mathrm{O}(2-1), \mathrm{DCN}(3-2), \mathrm{SiO}(5-4)$, and $\mathrm{SO}\left(5_{6}-4_{5}\right)$ in the E230 band. In this paper, we present the results of the ${ }^{13} \mathrm{CO}(2-1 ; 220.398684 \mathrm{GHz})$ and $\mathrm{C}^{18} \mathrm{O}(2-1$; $219.560358 \mathrm{GHz}$ ) lines. The main beam efficiency of the IRAM $30 \mathrm{~m}$ telescope at the frequency of $220 \mathrm{GHz}$ is 0.61 . The achieved velocity resolution at the frequencies of the ${ }^{13} \mathrm{CO}(2-1)$ and $\mathrm{C}^{18} \mathrm{O}$ (2-1) lines is $0.07 \mathrm{~km} \mathrm{~s}^{-1}$. The data were reduced with CLASS ${ }^{2}$. The achieved noise level in main beam temperature is $0.2-0.3 \mathrm{~K}$ at a velocity resolution of $0.07 \mathrm{~km} \mathrm{~s}^{-1}$ in the E230 band.

\subsection{ACA 7-m array observations}

Our observation toward HL Tau with the ACA 7-m array is a part of our ALMA project, 2015.1.00551.S. The observations were conducted on January 17, June 16, and September 8 in 2016. A 7-pointing mosaic pattern with the separation between each pointing of $20^{\prime \prime}$ was adopted, and the mapping area is larger than the field of view of the ALMA observations with the 12-m array (Yen et al. 2017). The total on-source time per pointing was $20 \mathrm{~min}$. On January 17, J0510+1800 (3.4 Jy at $220 \mathrm{GHz}$ ) was observed as flux and bandpass calibrators, and $\mathrm{J} 0431+1731(1.2-1.3 \mathrm{Jy}$ at $220 \mathrm{GHz})$ as a phase calibrator. On June 16 and September 9, the flux calibrators were Uranus and J0522-3627 (3.4 Jy at $220 \mathrm{GHz}$ ), respectively, and J0522-3627 was also observed as a bandpass calibrator. On these two days, J0510+1800 was observed as a phase calibrator (2.1 Jy on June 16 and $1.4 \mathrm{Jy}$ on September 9 at $220 \mathrm{GHz}$ ). The spectral setup of our ACA observations is the same as that of our observations with the 12-m array, which contains five spectral windows. Two spectral windows, each with a bandwidth of $2 \mathrm{GHz}$, were assigned to the $1.3 \mathrm{~mm}$ continuum. One spectral window with a bandwidth of $468.8 \mathrm{MHz}\left(638 \mathrm{~km} \mathrm{~s}^{-1}\right)$ and a channel width of $122 \mathrm{kHz}\left(0.17 \mathrm{~km} \mathrm{~s}^{-1}\right)$ was set to the ${ }^{13} \mathrm{CO}(2-1)$ line, one with a bandwidth of $234.4 \mathrm{MHz}\left(320 \mathrm{~km} \mathrm{~s}^{-1}\right)$ and a channel width of $244 \mathrm{kHz}\left(0.33 \mathrm{~km} \mathrm{~s}^{-1}\right)$ to the $\mathrm{C}^{18} \mathrm{O}$ (2-1) line, and one with a bandwidth of $234.4 \mathrm{MHz}\left(319 \mathrm{~km} \mathrm{~s}^{-1}\right)$ and a channel width of $488 \mathrm{kHz}\left(0.67 \mathrm{~km} \mathrm{~s}^{-1}\right)$ to the $\mathrm{SO}\left(5_{6}-4_{5}\right)$ line. The data were reduced with the pipeline in Common Astronomy Software Applications (CASA; McMullin et al. 2007) of version 4.7.0-1. The ACA 7-m array data were combined with the ALMA 12-m array data to generate images.

\subsection{Combined maps}

We generated combined images of the ${ }^{13} \mathrm{CO}(2-1)$ and $\mathrm{C}^{18} \mathrm{O}$ (2-1) emission in HL Tau with the IRAM 30m, ACA, and ALMA data using CASA of version 4.7.0. The details of our ALMA observations were described in Yen et al. (2017). We first adopted imagermode of "mosaic" and generated interferometric images with the ACA and ALMA data together using the CASA task, clean. These interferometric images of the ${ }^{13} \mathrm{CO}(2-1)$ and $\mathrm{C}^{18} \mathrm{O}(2-1)$ emission were generated with the natural weighting at velocity resolutions of 0.2 and $0.34 \mathrm{~km} \mathrm{~s}^{-1}$, respectively. Multiscale clean with the scale sizes of $0,2^{\prime \prime}, 5^{\prime \prime}$, and $10^{\prime \prime}$ was performed. Then, we regrided the IRAM $30 \mathrm{~m}$ images to have the same velocity axis as the interferometric images and multiplied them by the primary beam response of the mosaic observations. Finally, we combined the interferometric and IRAM 30m images to generate the combined images using the CASA task, feather, and its visual interface, casafeather. The final resolutions of the combined images are $1^{\prime \prime}$, and the noise levels are 6 and $8 \mathrm{mJy}$ beam ${ }^{-1}$ in the ${ }^{13} \mathrm{CO}(2-1)$ and $\mathrm{C}^{18} \mathrm{O}(2-1)$ images, respectively

\footnotetext{
2 http://www.iram.fr/IRAMFR/GILDAS
}

(Table 1). We note that the images from our mosaicking observations with the 7-m array are affected by the imaging issues of $\mathrm{CASA}^{3}$, and the fluxes in our mosaic map are overestimated by $10 \%$. Nevertheless, in this work, we analyze the intensity distributions in the velocity channel maps, and our analysis does not depend on the flux level. Thus, our discussions and conclusions are not affected by this imaging issue.

\section{Results}

\subsection{Single-dish maps}

Figure 1 presents the total-integrated intensity (moment 0 ) maps of the ${ }^{13} \mathrm{CO}$ and $\mathrm{C}^{18} \mathrm{O}$ emission lines in HL Tau. The intensity distributions of the ${ }^{13} \mathrm{CO}$ lines are centrally peaked at the position of HL Tau. In contrast, the $\mathrm{C}^{18} \mathrm{O}$ lines show a filamentary structure from the northwest to the south of HL Tau, and there is no clear intensity enhancement at the position of HL Tau. Two $\mathrm{T}$ Tauri stars, XZ Tau and $\mathrm{LkH} \alpha 358$, are located outside the regions exhibiting the intense ${ }^{13} \mathrm{CO}$ and $\mathrm{C}^{18} \mathrm{O}$ emission, suggesting that they are not associated with dense molecular gas on a scale of thousands of au.

Figure 2 presents the ${ }^{13} \mathrm{CO}$ and $\mathrm{C}^{18} \mathrm{O}$ spectra at nine different positions. To compare the spectra of the different emission lines, all the data have been first convolved with the same angular resolution of $155^{\prime \prime} 3$ and re-gridded to have the same pixel size and channel width. The systemic velocity of the circumstellar disk around HL Tau is measured to be $V_{\mathrm{LSR}}$ of $7 \mathrm{~km} \mathrm{~s}^{-1}$ from the Keplerian rotation of the $\operatorname{disk}^{4}$ (Yen et al. 2017), and we adopt this velocity as the systemic velocity of HL Tau. The velocity of the intensity peak $\left(V_{\text {peak }}\right)$ of the ${ }^{13} \mathrm{CO}$ emission at the position of HL Tau is $6.4 \mathrm{~km} \mathrm{~s}^{-1}$, which is offset from the systemic velocity of HL Tau (Fig. 2). As shown in these spectra, the majority of the emission observed with the single dishes, tracing the largescale gas and the envelope around HL Tau, is at the velocities more blueshifted with respect to the systemic velocity of HL Tau.

The intensity ratio of ${ }^{13} \mathrm{CO}(3-2)$ to $(2-1)$ at the position of HL Tau clearly increases as the relative velocity with respect to $V_{\text {peak }}$ increases. The ${ }^{13} \mathrm{CO}(3-2)$ to $(2-1)$ intensity ratio is $\sim 0.7$ at $V_{\text {peak }}$, and the intensity ratio becomes close to or larger than unity at the velocities of $V_{\mathrm{LSR}} \lesssim 5.6 \mathrm{~km} \mathrm{~s}^{-1}$ and $V_{\mathrm{LSR}} \gtrsim 7.2 \mathrm{~km} \mathrm{~s}^{-1}$. Similar trends are also seen in the spectra at the other positions, for example, at the blueshifted velocity at $\left(30^{\prime \prime}, 30^{\prime \prime}\right)$ and $\left(30^{\prime \prime}, 0^{\prime \prime}\right)$ and at the redshifted velocity at $\left(-30^{\prime \prime},-30^{\prime \prime}\right)$. In addition, these spectra show that the $\mathrm{C}^{18} \mathrm{O}$ line wing emission at these higher velocities with respect to $V_{\text {peak }}$, where the ${ }^{13} \mathrm{CO}(3-2)$ to (2-1) intensity ratio is higher, is below the detection level of our observations. This change in the intensity ratio of the ${ }^{13} \mathrm{CO}$ lines suggests that the physical conditions at $V_{\text {peak }}$ and those traced by the line wing emission are different.

On the assumption of the local thermal equilibrium (LTE) condition, the excitation temperature $T_{\mathrm{ex}}$ and optical depths $(\tau)$ of the ${ }^{13} \mathrm{CO}(3-2)$ and (2-1) lines can be estimated from

$\frac{T_{1}}{T_{2}}=\left(\frac{B_{v_{1}}\left(T_{\mathrm{ex}}\right)-B_{v_{1}}\left(T_{\mathrm{bg}}\right)}{B_{v_{2}}\left(T_{\mathrm{ex}}\right)-B_{v_{2}}\left(T_{\mathrm{bg}}\right)}\right)\left(\frac{1-e^{-\tau_{1}}}{1-e^{-\tau_{2}}}\right)$,

3 http://library.nrao.edu/public/memos/naasc/NAASC_ 117.pdf

4 To measure the systemic velocity, the peak positions in different velocity channels were measured from the position-velocity diagram along the disk major axis of the ${ }^{13} \mathrm{CO}(2-1)$ and $\mathrm{C}^{18} \mathrm{O}(2-1)$ emission observed with ALMA at 0. ' 8 resolutions, and a radial velocity profile of Keplerian rotation with two free parameters - stellar mass and systemic velocity - was fitted to these data points. 

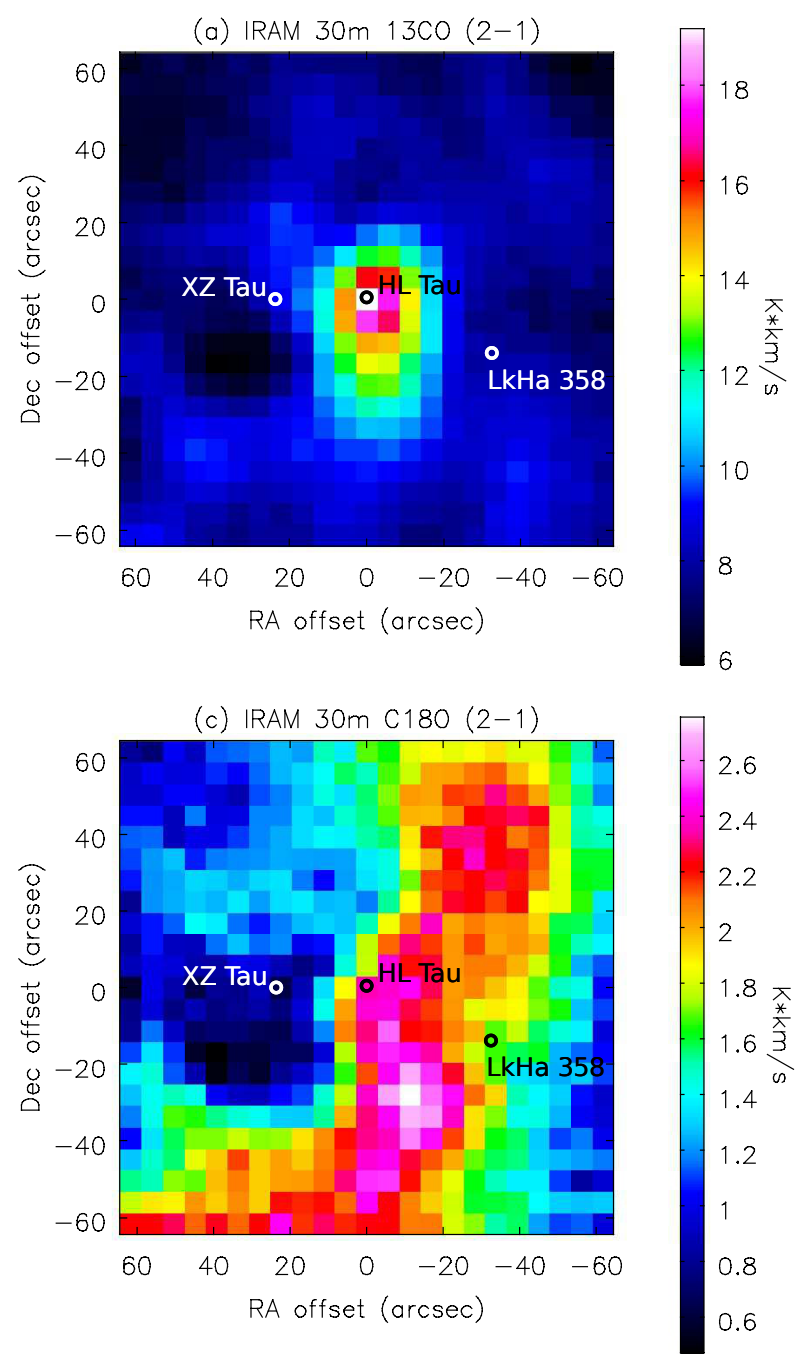
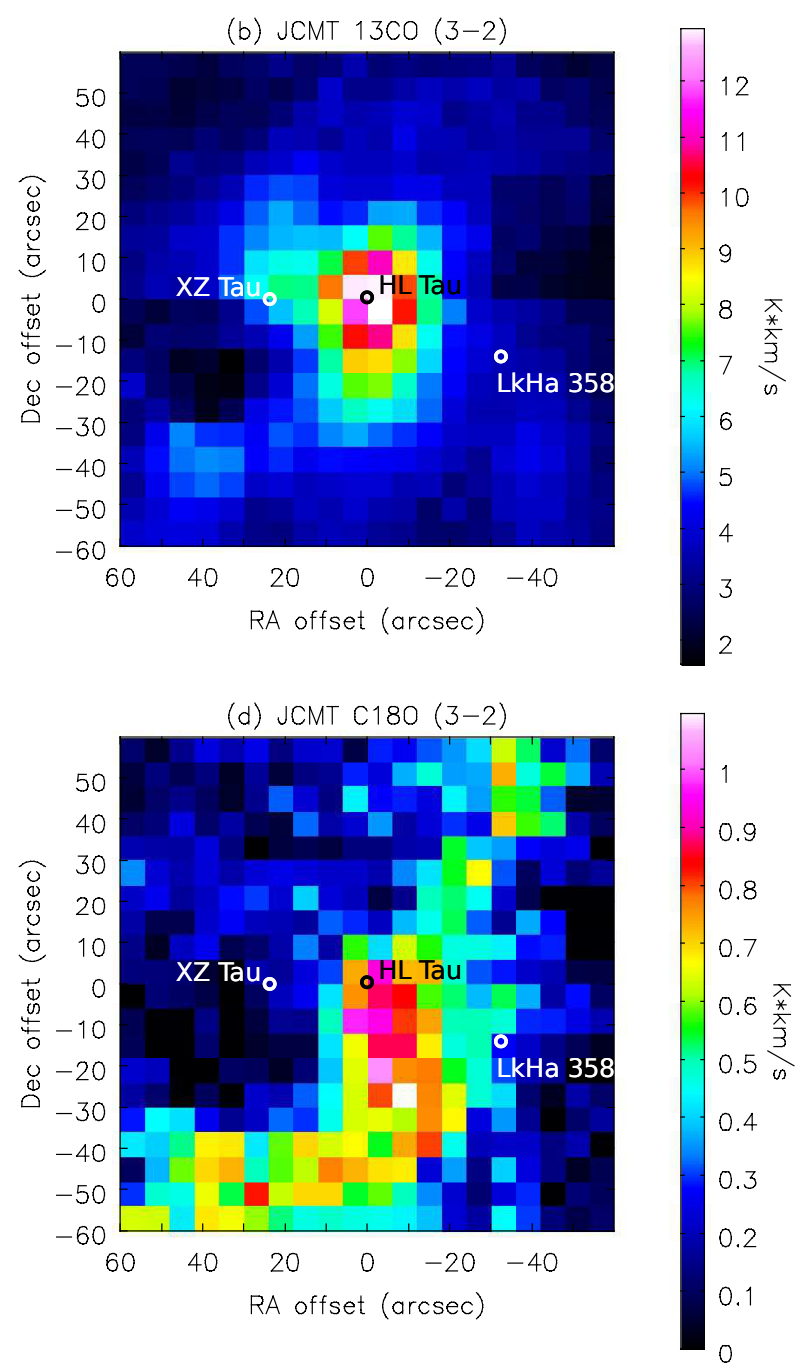

Fig. 1. Moment 0 maps of the ${ }^{13} \mathrm{CO}(2-1)$ and $\mathrm{C}^{18} \mathrm{O}(2-1)$ emission (panels $a$ and $c$ ) obtained with the IRAM $30 \mathrm{~m}$ telescope and the ${ }^{13} \mathrm{CO}(3-2)$ and $\mathrm{C}^{18} \mathrm{O}(3-2)$ emission (panels $b$ and $d$ ) obtained with JCMT.

where $B_{v}(T)$ is the Planck function at the frequency $v$ and the temperature $T$, and $T_{\text {bg }}$ is the cosmic background temperature of $2.73 \mathrm{~K}$. Here, $T_{1}, v_{1}$, and $\tau_{1}$ are the brightness temperature, frequency, and optical depth of the ${ }^{13} \mathrm{CO}(2-1)$ line, and $T_{2}, v_{2}$, and $\tau_{2}$ are those of the ${ }^{13} \mathrm{CO}(3-2)$ line. We assumed that the ${ }^{13} \mathrm{CO}(2-1)$ and (3-2) lines have the same $T_{\mathrm{ex}}$. With a given $T_{\mathrm{ex}}$, the ratio of $\tau_{1}$ to $\tau_{2}$ can be computed following the equations in Mangum \& Shirley (2015). Then, the number of unknowns is reduced to two in Eq. (1), which are $T_{\mathrm{ex}}$ and $\tau_{1}$. Thus, $T_{\mathrm{ex}}, \tau_{1}$, and $\tau_{2}$ can be derived with Eq. (1) from the brightness temperature of the ${ }^{13} \mathrm{CO}(2-1)$ and (3-2) lines.

At the position of HL Tau, the brightness temperatures of the ${ }^{13} \mathrm{CO}(2-1)$ and (3-2) lines are both $2.4 \mathrm{~K}$ at $V_{\mathrm{LSR}}$ of $5.4 \mathrm{~km} \mathrm{~s}^{-1}$ and $5 \mathrm{~K}$ at $V_{\mathrm{LSR}}$ of $7.3 \mathrm{~km} \mathrm{~s}^{-1}$. With Eq. (1), $T_{\mathrm{ex}}$ is estimated to be $25 \pm 9 \mathrm{~K}$ with both $\tau$ of $0.1 \pm 0.1$ at $V_{\mathrm{LSR}}$ of $5.4 \mathrm{~km} \mathrm{~s}^{-1}$. At $V_{\mathrm{LSR}}$ of $7.3 \mathrm{~km} \mathrm{~s}^{-1}, T_{\text {ex }}$ is estimated to be $27 \pm 6 \mathrm{~K}$, and both $\tau$ are $0.3 \pm 0.1$. The uncertainties are estimated from the error propagation of the observational noise. At these higher velocities with respect to $V_{\text {peak }}$, where the intensity ratio is higher, the ${ }^{13} \mathrm{CO}$ lines are optically thin. $\mathrm{C}^{18} \mathrm{O}$ is less abundant than ${ }^{13} \mathrm{CO}$ with a ${ }^{13} \mathrm{CO} / \mathrm{C}^{18} \mathrm{O}$ abundance ratio of $5.5-10$ in HL Tau (Wilson \& Rood 1994; Brittain et al. 2005; Smith et al. 2015). Thus, the $\mathrm{C}^{18} \mathrm{O}$ lines are expected also to be optically thin at these higher velocities. In this case, the ratio of the brightness temperature between the ${ }^{13} \mathrm{CO}$ and $\mathrm{C}^{18} \mathrm{O}$ lines is approximately their abundance ratio, and the expected $\mathrm{C}^{18} \mathrm{O}$ brightness temperature at the higher velocities with respect to $V_{\text {peak }}$ is lower than the noise levels in our observations.

As shown in Fig. 2, the ${ }^{13} \mathrm{CO}(2-1)$ line is brighter than the ${ }^{13} \mathrm{CO}(3-2)$ line at the velocity around $V_{\text {peak }}$, for example, at $V_{\mathrm{LSR}}=5.6-7.2 \mathrm{~km} \mathrm{~s}^{-1}$ at the position of HL Tau. The ${ }^{13} \mathrm{CO}$ (3$2) /(2-1)$ intensity ratio is expected to decrease with decreasing temperature (Eq. (1)). Thus, the gas temperature in the HL Tau region is expected to be lower at the velocity around $V_{\text {peak }}$. At $V_{\text {peak }}$, which is $V_{\mathrm{LSR}}$ of $6.4 \mathrm{~km} \mathrm{~s}^{-1}$, the brightness temperatures of the ${ }^{13} \mathrm{CO}(2-1)$ and (3-2) lines are 8.9 and $5.7 \mathrm{~K}$, respectively. With Eq. (1), $T_{\mathrm{ex}}$ is estimated to be $14 \pm 1 \mathrm{~K}$, and $\tau$ of the ${ }^{13} \mathrm{CO}(2-1)$ and (3-2) lines are estimated to be $1.5 \pm 0.2$ and $1.4 \pm 0.2$, respectively. Here, the signal-to-noise ratios $(\mathrm{S} / \mathrm{Ns})$ of the emission lines are higher than ten, and thus, the dominant source of the uncertainty in the estimate is the absolute flux uncertainties of the IRAM 30m telescope and JCMT, which are $10 \%$. Similarly, from the intensity ratio of the $\mathrm{C}^{18} \mathrm{O}(3-2)$ to (21) lines, 1.4 over $1.9 \mathrm{~K}$, at the velocity of the $\mathrm{C}^{18} \mathrm{O}$ intensity peak, $V_{\mathrm{LSR}}$ of $6.7 \mathrm{~km} \mathrm{~s}^{-1}, T_{\mathrm{ex}}$ is estimated to be $15 \pm 7 \mathrm{~K}$, and $\tau$ of the $\mathrm{C}^{18} \mathrm{O}(2-1)$ and (3-2) lines are estimated to be $0.3 \pm 0.3$ and 


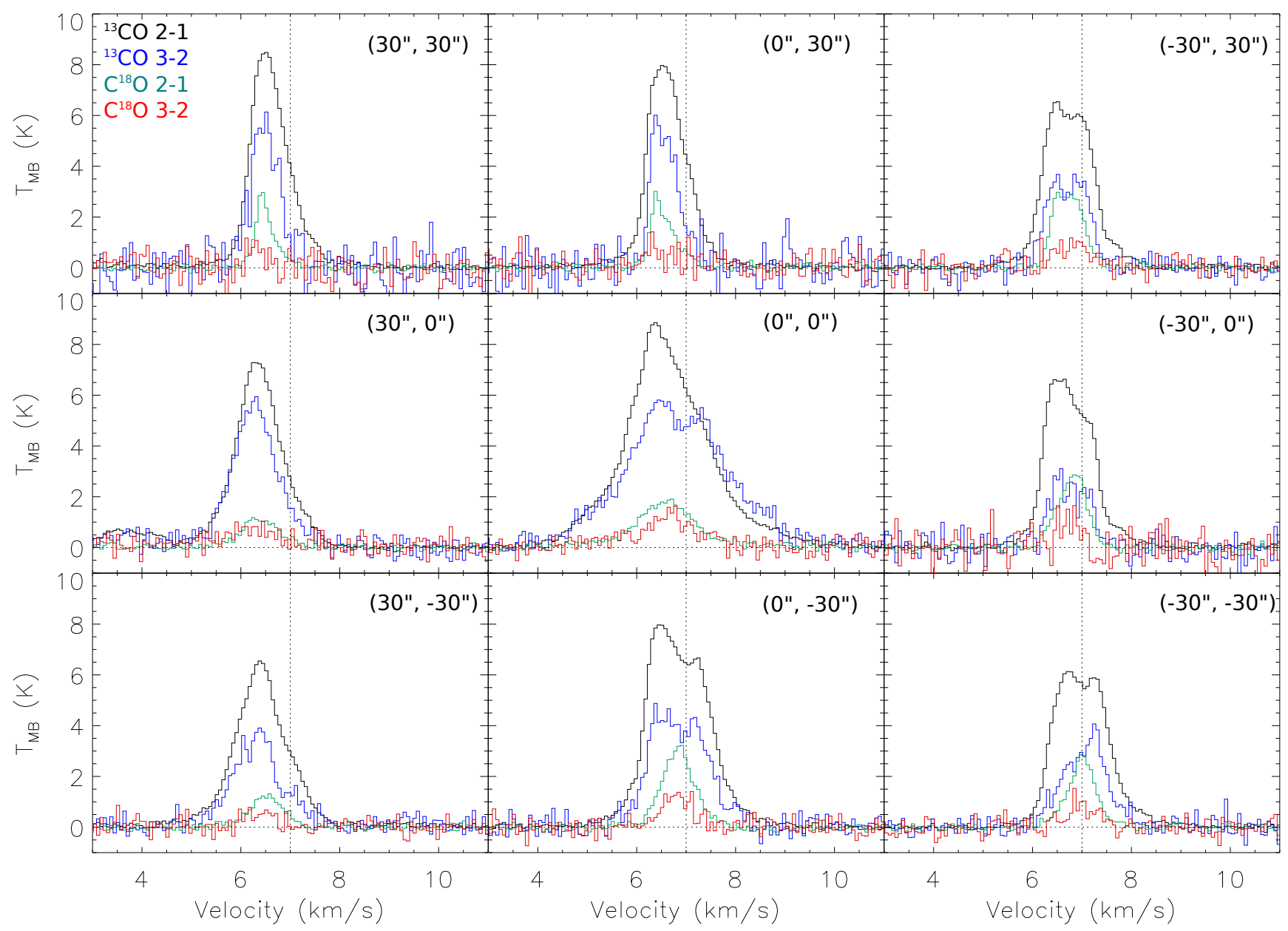

Fig. 2. Spectra of the ${ }^{13} \mathrm{CO}\left(2-1\right.$; black histograms) and (3-2; blue histograms) emission and the $\mathrm{C}^{18} \mathrm{O}(2-1$; green histograms) and (3-2; red histograms) emission at nine different positions in the HL Tau region obtained with our single-dish observations. The position at which the spectra were extracted is labeled at the upper right corner in each panel in relative offset with respect to the position of HL Tau. Vertical dashed lines denote the systemic velocity of HL Tau, $V_{\mathrm{LSR}}=7 \mathrm{~km} \mathrm{~s}^{-1}$.

$0.2 \pm 0.2$, respectively. Therefore, these results show that around HL Tau there are relatively warmer gas with a lower column density traced by the line wing emission at the higher velocities with respect to the peak velocity, where the lines are all optically thin, and relatively cooler gas with a higher column density at the velocity close to the peak velocity, where the ${ }^{13} \mathrm{CO}$ lines are optically thick and the $\mathrm{C}^{18} \mathrm{O}$ lines are optically thin.

Figure 3 presents the moment 0 maps integrated over different velocity ranges to show the gas distributions at the higher and lower velocities with respect to $V_{\text {peak }}$. Blue and red contours show the emission at the relative velocities of $>0.4-0.5 \mathrm{~km} \mathrm{~s}^{-1}$ with respect to $V_{\text {peak }}$, corresponding to $V_{\mathrm{LSR}}<6.2 \mathrm{~km} \mathrm{~s}^{-1}$ and $V_{\mathrm{LSR}}>7.1 \mathrm{~km} \mathrm{~s}^{-1}$, respectively, and the grayscales show the emission at the velocity close to $V_{\text {peak }}, V_{\mathrm{LSR}}=6.2-7.1 \mathrm{~km} \mathrm{~s}^{-1}$. The $\mathrm{C}^{18} \mathrm{O}$ emission at the velocity close to $V_{\text {peak }}$ is elongated from the northwest to the south, identical to the total-integrated intensity (Fig. 1). Similar north-to-south elongation can also be seen in the ${ }^{13} \mathrm{CO}$ emission at the lower velocities. At the higher relative velocities with respect to $V_{\text {peak }}$, the ${ }^{13} \mathrm{CO}$ (3-2) and $\mathrm{C}^{18} \mathrm{O}(2-1)$ lines show that the blueshifted emission is located in the northeast and extends toward the east, and that the redshifted emission is located in the southwest and extends toward the southwest. A velocity gradient along the same direction, from the northeast to the southwest, is also observed in the ${ }^{13} \mathrm{CO}(2-1)$ emission at these velocities. The $\mathrm{C}^{18} \mathrm{O}(3-2)$ emission is not clearly detected at $V_{\mathrm{LSR}}<6.2 \mathrm{~km} \mathrm{~s}^{-1}$ and $V_{\mathrm{LSR}}>7.1 \mathrm{~km} \mathrm{~s}^{-1}$. The direction of the velocity gradient observed in the ${ }^{13} \mathrm{CO}(3-2)$ and $(2-1)$ and $\mathrm{C}^{18} \mathrm{O}(2-1)$ emission at the higher velocities are consistent with that of the bipolar molecular outflow associated with HL Tau (Lumbreras \& Zapata 2014; ALMA Partnership 2015; Klaassen et al. 2016). These results suggest that the emission lines at $V_{\mathrm{LSR}}<6.2 \mathrm{~km} \mathrm{~s}^{-1}$ and $V_{\mathrm{LSR}}>7.1 \mathrm{~km} \mathrm{~s}^{-1}$ on a largescale of $\sim 5000$ au observed with the single dishes likely have the contribution from the outflow, and that the $\mathrm{C}^{18} \mathrm{O}$ lines are less contaminated by the outflow and better trace the density distribution of the ambient cooler gas with a higher column density around HL Tau, compared to the ${ }^{13} \mathrm{CO}$ lines. In addition, the northwest-south elongation of the $\mathrm{C}^{18} \mathrm{O}$ emission is most likely associated with the western edge of the $0.1 \mathrm{pc}$ shell-like structure observed in the ${ }^{13} \mathrm{CO}(1-0)$ emission with the combined data of the NRAO $12 \mathrm{~m}$ telescope and the BIMA array (Welch et al. 2000).

\subsection{Combined maps}

Figures 4 and 5 present the moment 0 maps of the ${ }^{13} \mathrm{CO}(2-1)$ and $\mathrm{C}^{18} \mathrm{O}(2-1)$ emission integrated over different velocity ranges in HL Tau obtained by combining the IRAM 30m, ACA, and ALMA data. The integrated velocity ranges for the ${ }^{13} \mathrm{CO}$ line are high velocities of $V_{\mathrm{LSR}}=1-4$ and $8.6-13.4 \mathrm{~km} \mathrm{~s}^{-1}$, medium 

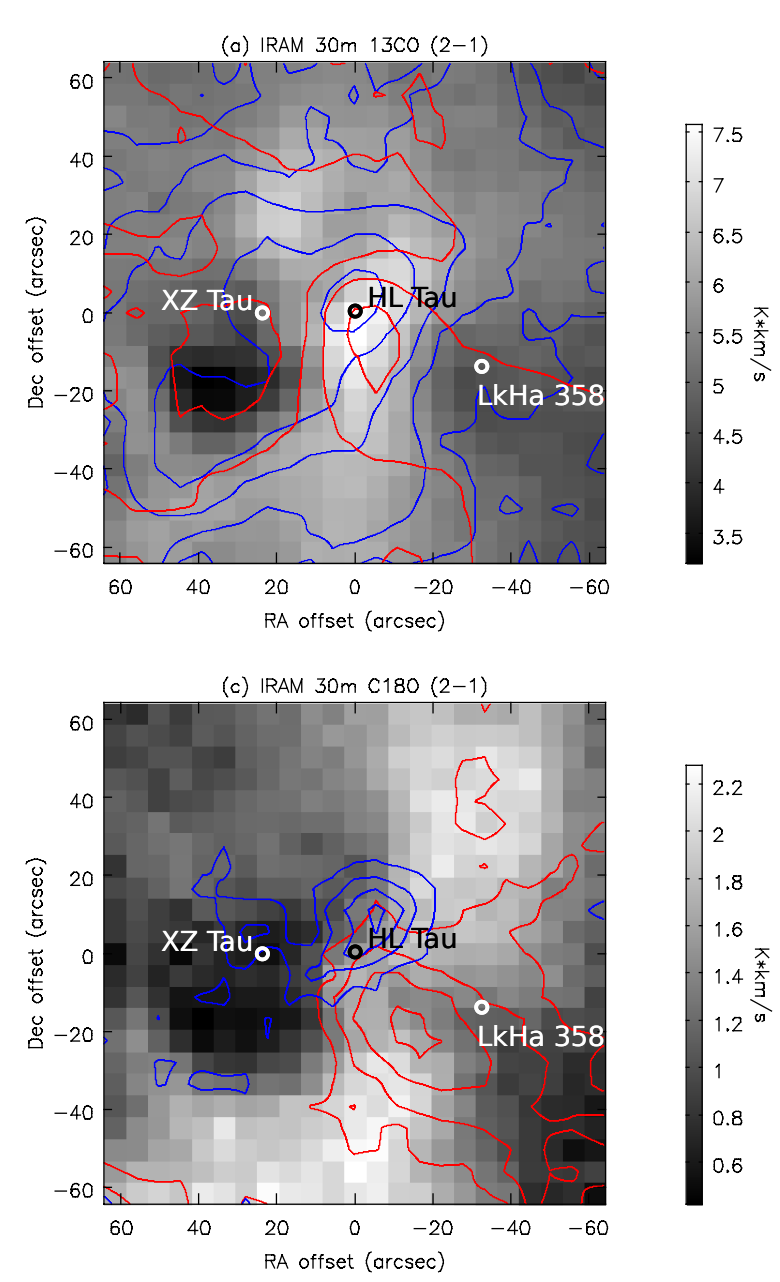
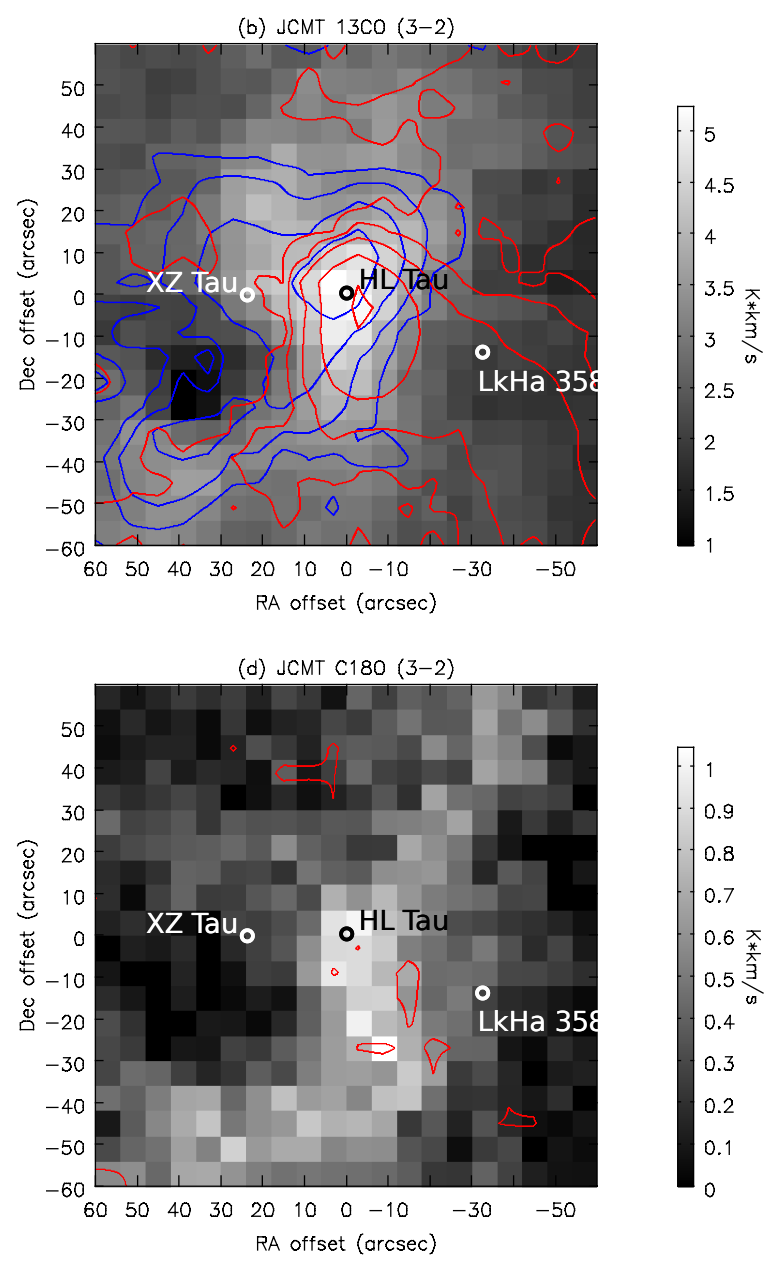

Fig. 3. Single-dish moment 0 maps of the ${ }^{13} \mathrm{CO}(2-1)$ and (3-2) emission (panels a and $b$ ) and the $\mathrm{C}^{18} \mathrm{O}(2-1)$ and (3-2) emission (panels $c$ and $d$ ) integrated over different velocity ranges. Grayscales present the integrated intensity in the velocity range close to the velocity of the intensity peak $V_{\mathrm{LSR}}=6.2-7.1 \mathrm{~km} \mathrm{~s}^{-1}$. Blue and red contours present the integrated intensity at the higher relative velocities with respect to the peak velocity, $V_{\mathrm{LSR}}<6.2 \mathrm{~km} \mathrm{~s}^{-1}$ and $V_{\mathrm{LSR}}>7.1 \mathrm{~km} \mathrm{~s}^{-1}$, respectively. Contours are from $3 \sigma$ and increase in steps of a factor of two. $1 \sigma$ levels of the integrated blue- and redshifted emission are 0.09 and $0.11 \mathrm{~K} \mathrm{~km} \mathrm{~s}^{-1}$ in panel $a, 0.11$ and $0.1 \mathrm{~K} \mathrm{~km} \mathrm{~s}^{-1}$ in panel b, and 0.04 and $0.07 \mathrm{~K} \mathrm{~km} \mathrm{~s}^{-1}$ in panel $c$. In the $\mathrm{C}^{18} \mathrm{O}(3-2)$ line, there is no clear emission detected at the blueshifted high velocity, and the $1 \sigma$ level of the integrated redshifted emission is $0.1 \mathrm{~K} \mathrm{~km} \mathrm{~s}^{-1}$.

velocities of $V_{\mathrm{LSR}}=4-5.6$ and $7.8-8.6 \mathrm{~km} \mathrm{~s}^{-1}$, and low velocities of $V_{\mathrm{LSR}}=5.6-7$ and $7-7.8 \mathrm{~km} \mathrm{~s}^{-1}$. Those for the $\mathrm{C}^{18} \mathrm{O}$ line are high velocities of $V_{\mathrm{LSR}}=3-4.7$ and $8.5-12 \mathrm{~km} \mathrm{~s}^{-1}$, medium velocities of $V_{\mathrm{LSR}}=4.7-5.4$ and $7.8-8.5 \mathrm{~km} \mathrm{~s}^{-1}$, and low velocities of $V_{\mathrm{LSR}}=5.4-7.1$ and $7.1-7.8 \mathrm{~km} \mathrm{~s}^{-1}$. These combined maps do not suffer from the effect of the missing flux, different from the ALMA maps of the ${ }^{13} \mathrm{CO}(2-1)$ and $\mathrm{C}^{18} \mathrm{O}(2-1)$ emission in Yen et al. (2017).

As discussed in Yen et al. (2017), the high-velocity ${ }^{13} \mathrm{CO}$ and $\mathrm{C}^{18} \mathrm{O}$ components primarily trace the Keplerian rotation of the disk with a radius of $\sim 100$ au around HL Tau. After adding the short-spacing data, a possible contamination from the outflow is seen in the redshifted high-velocity ${ }^{13} \mathrm{CO}$ emission, showing a fan-shape structure extending toward the southwest. Such an outflow contamination is not seen in the $\mathrm{C}^{18} \mathrm{O}$ emission. At the medium velocities, two arc-like structures are observed in both the ${ }^{13} \mathrm{CO}$ and $\mathrm{C}^{18} \mathrm{O}$ emission. One arc-like structure is blueshifted, and the other is redshifted. The blueshifted arc-like structure has a length of 2000 au and stretches from the east to the northwest. The redshifted arc-like structure has a length of $1000 \mathrm{au}$ and stretches from the west to the southeast. In addition, in our combined maps, the extended emission attached to the arc-like structures is also observed in the ${ }^{13} \mathrm{CO}$ emission, and the redshifted arc-like structure becomes less evident, compared to the ALMA map in Yen et al. (2017). At the low velocities, the ${ }^{13} \mathrm{CO}$ emission is detected over the entire field of the view. For clarity, in Figs. 4 and 5 we plot only the contours of the integrated intensity that are above $20 \sigma$. At the medium and low velocities, the ${ }^{13} \mathrm{CO}$ emission exhibits a clear velocity gradient along the northeast-southwest direction, where the northeastern part is blueshifted and the southwestern part is redshifted. A similar velocity gradient is also observed in the $\mathrm{C}^{18} \mathrm{O}$ emission at the low velocities. The redshifted $\mathrm{C}^{18} \mathrm{O}$ emission at the low velocities additionally shows a fanshaped structure extending toward the southwest with its apex located at the protostellar position. This morphology is similar to the outflow in HL Tau observed in the CO (1-0) emission with ALMA (Klaassen et al. 2016) and in the CO (3-2) emission with SMA (Lumbreras \& Zapata 2014). Therefore, at the low velocities, there is a possible contamination from the outflow.

With the combined data, the arc-like structures, which were only detected in the ${ }^{13} \mathrm{CO}$ emission at the medium velocities in Yen et al. (2017), are now also detected in the $\mathrm{C}^{18} \mathrm{O}$ emission 
(a) high velocity

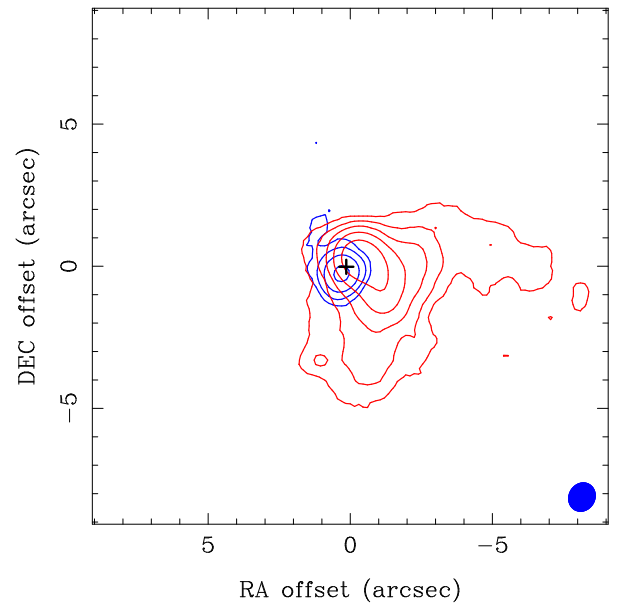

(b) medium velocity

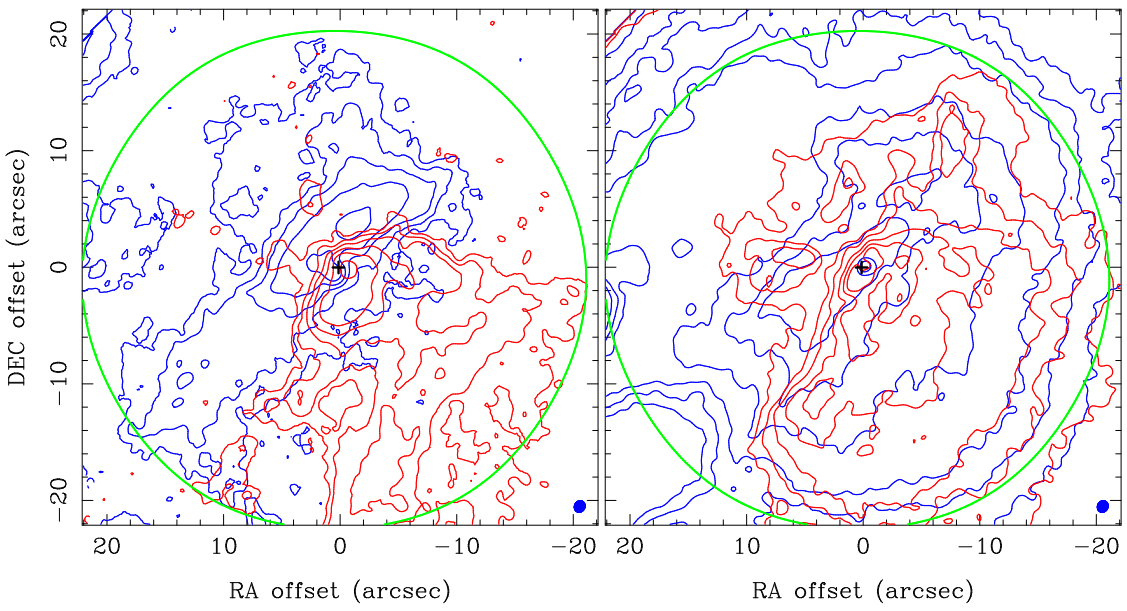

Fig. 4. Moment 0 maps of the ${ }^{13} \mathrm{CO}(2-1)$ emission integrated over the different velocity ranges from the combined data obtained with the IRAM $30 \mathrm{~m}$, ACA, and ALMA observations. The integrated velocity ranges are high velocities of $V_{\mathrm{LSR}}=3-4.7$ and $8.5-12 \mathrm{~km} \mathrm{~s}^{-1}$, medium velocities of $V_{\mathrm{LSR}}=4.7-5.4$ and 7.8-8.5 $\mathrm{km} \mathrm{s}^{-1}$, and low velocities of $V_{\mathrm{LSR}}=5.4-7.1$ and 7.1-7.8 $\mathrm{km} \mathrm{s}^{-1}$. Blue and red contours show the blue- and redshifted emission, respectively. Contour levels are from $5 \sigma$ and increase in steps of a factor of two in panels $a$ and $b$, and those are $20 \sigma, 30 \sigma, 40 \sigma$, and then in steps of $20 \sigma$ in panel c. $1 \sigma$ levels of the integrated blue- and redshifted emission are 6 and $7.4 \mathrm{mJy} \mathrm{beam}^{-1} \mathrm{~km} \mathrm{~s}^{-1}$ in panel $a, 4.6$ and $3.4 \mathrm{mJy}$ beam ${ }^{-1} \mathrm{~km} \mathrm{~s}^{-1}$ in panel $b$, and 3.8 and $3.1 \mathrm{mJy} \mathrm{beam}^{-1} \mathrm{~km} \mathrm{~s}^{-1}$ in panel $c$. Crosses denote the position of HL Tau. Filled blue ellipses present the size of the synthesized beam. Green circles show the region where the primary beam response is 0.5 .

(a) high velocity

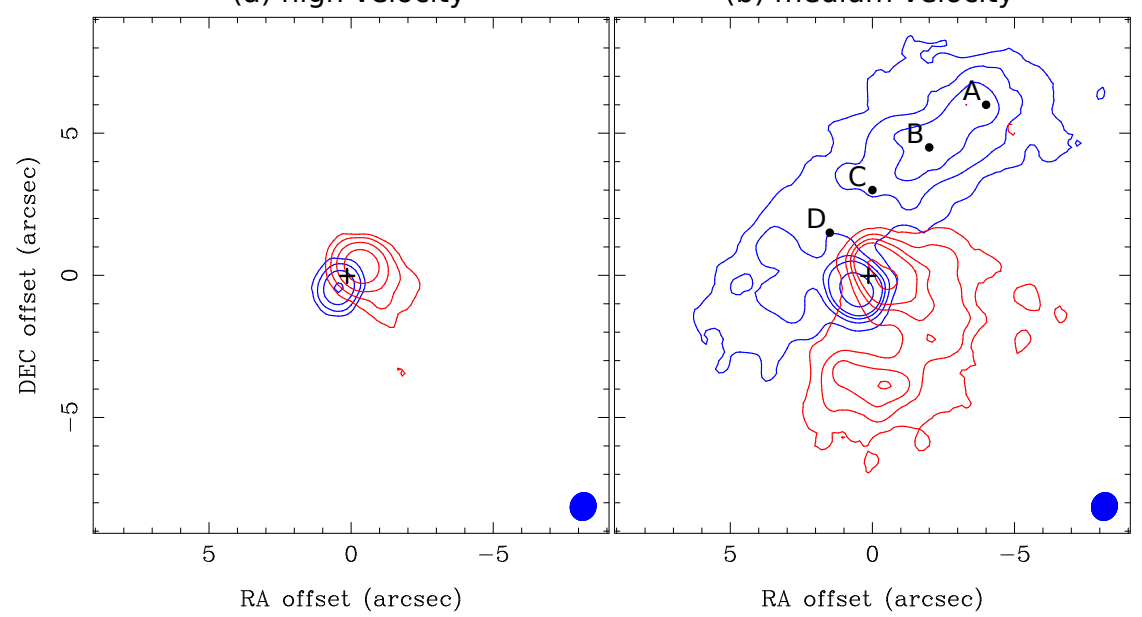

(c) low velocity

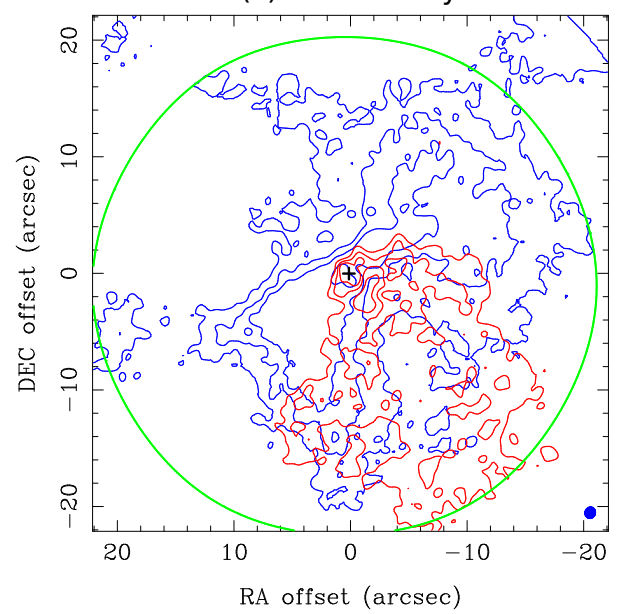

Fig. 5. Same as Fig. 4 but for the $\mathrm{C}^{18} \mathrm{O}(2-1)$ emission. The integrated velocity ranges are high velocities of $V_{\mathrm{LSR}}=1-4$ and $8.6-13.4 \mathrm{~km} \mathrm{~s}{ }^{-1}$, medium velocities of $V_{\mathrm{LSR}}=4-5.6$ and $7.8-8.6 \mathrm{~km} \mathrm{~s}^{-1}$, and low velocities of $V_{\mathrm{LSR}}=5.6-7$ and 7-7.8 $\mathrm{km} \mathrm{s}^{-1}$. Contour levels are from 5 $\sigma$ and increase in steps of a factor two in panels $a$ and $b$, and those are $10 \sigma, 15 \sigma, 20 \sigma, 40 \sigma$, and $80 \sigma$ in panel c. $1 \sigma$ levels of the integrated blue- and redshifted emission are 4.2 and $5.9 \mathrm{mJy} \mathrm{beam}^{-1} \mathrm{~km} \mathrm{~s}^{-1}$ in panel $a$, both $3.2 \mathrm{mJy}$ beam ${ }^{-1} \mathrm{~km} \mathrm{~s}^{-1}$ in panel $b$, and 3.7 and $2.6 \mathrm{mJy} \mathrm{beam}^{-1} \mathrm{~km} \mathrm{~s}^{-1}$ in panel $c$. The positions to extract spectra, which are shown in Fig. 7, are labeled as A-D in panel $b$.

at similar velocities. The entire velocity channel maps in the velocity ranges of the medium and low velocities are shown in Fig. 6. As shown in Fig. 6, the arc-like structures are more compact and located closer to the protostar at a higher velocity. As the relative velocities with respect to the systemic velocity of HL Tau decrease, the extensions of the arc-like structures increase, and the arc-like structures gradually merge with the extended emission at the low velocities of $V_{\mathrm{LSR}}=6.1$ and $7.5 \mathrm{~km} \mathrm{~s}^{-1}$.

Two possible origins of the arc-like structures, infalling material from the extended envelope around HL Tau and mass ejection from the HL Tau disk, were discussed in Yen et al. (2017). Gaseous clumps ejected from a disk tend to remain compact as they move away from the disk, as shown in numerical simulations (e.g., Vorobyov 2016). Thus, the gradual change in the spatial structures from the small to largescales seen in our combined maps suggests that the arc-like structures are unlikely mass ejection from the disk. In addition, the arc-like structures show a higher velocity at a smaller radius. Such a radial velocity profile is opposite to that in structures swept up by outflows, which tend to show a higher velocity at a larger radius (Shu et al. 1991, 2000; Lee et al. 2000). The CO outflow in HL Tau also shows a higher velocity at a larger radius as observed with SMA (Lumbreras \& Zapata 2014). Therefore, the arc-like structures more likely originate from the extended envelope around HL Tau.

As discussed in Yen et al. (2017), the blueshifted arc-like structure exhibits relative velocities with respect to the systemic 


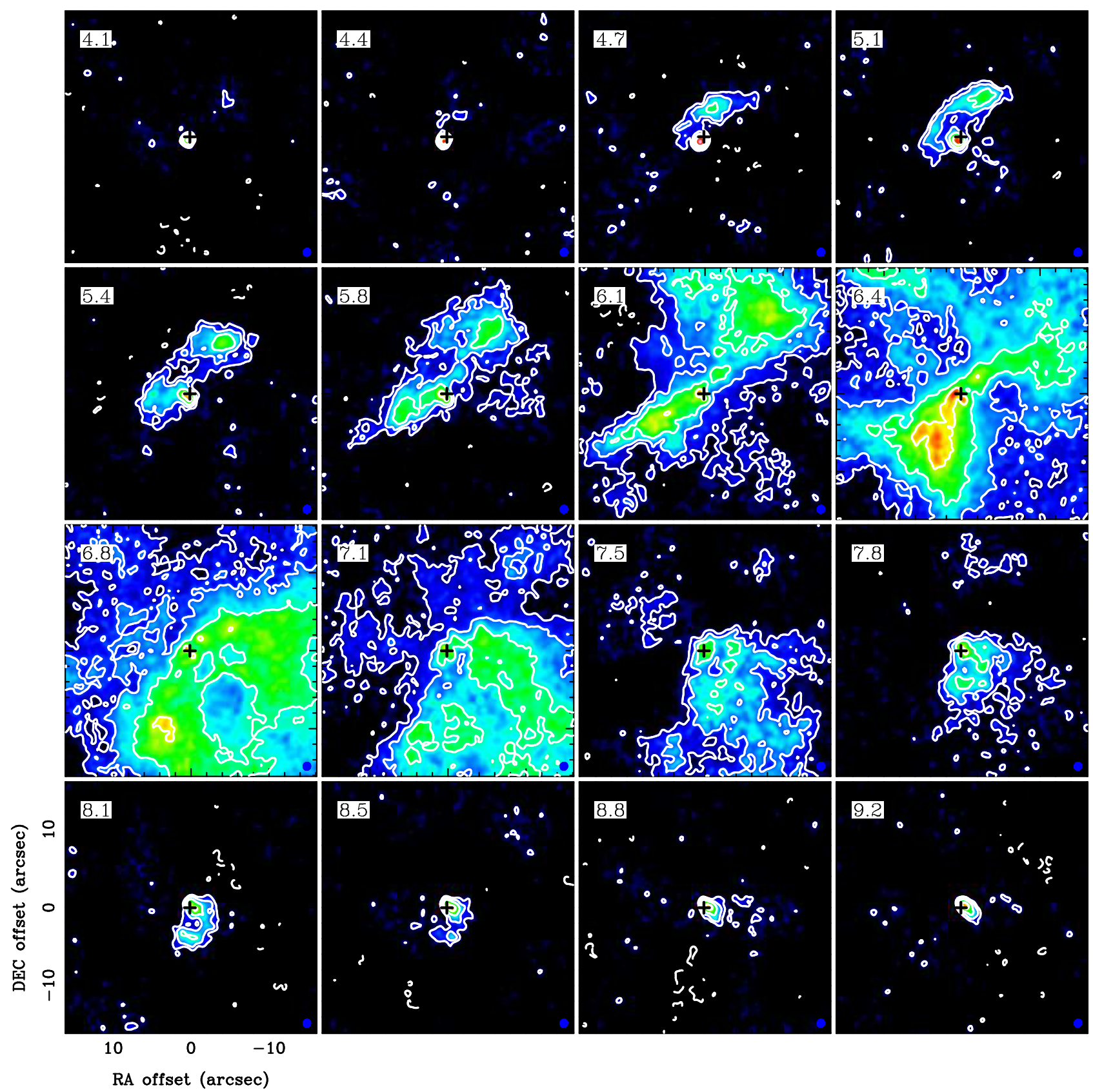

Fig. 6. Velocity channel maps of the $\mathrm{C}^{18} \mathrm{O}(2-1)$ emission at the medium and low velocities generated from the combined data obtained with the IRAM 30m, ACA, and ALMA observations. Contour levels are from $3 \sigma$ and increase in steps of a factor of two, where $1 \sigma$ is $5.5 \mathrm{mJy}^{\mathrm{B}} \mathrm{Beam}{ }^{-1}$. The central velocity of each channel is labeled at the upper left corner in each panel in units of $\mathrm{km} \mathrm{s}^{-1}$. Crosses denote the position of HL Tau. Blue filled ellipses present the size of the synthetized beam.

velocity of HL Tau higher than the expected free-fall velocities ${ }^{5}$, and there is a possibility that such a velocity excess is caused by the missing flux in the ALMA data and the absorption by the largescale dense cloud or outflow in the ${ }^{13} \mathrm{CO}$ emission, which is optically thick at the low velocities. The combined map of the $\mathrm{C}^{18} \mathrm{O}$ emission does not suffer from these effects because there is no missing flux and the $\mathrm{C}^{18} \mathrm{O}$ emission is

\footnotetext{
5 The expected free-fall velocity was computed with the central stellar mass of $1.8 M_{\odot}$ estimated from the Keplerian rotation observed in the $\mathrm{C}^{18} \mathrm{O}$ and ${ }^{13} \mathrm{CO}$ emission at a $0 .{ }^{\prime} 8$ resolution and the inclination angle of $47^{\circ}$ estimated from the orientation of the circumstellar disk observed in the $1 \mathrm{~mm}$ continuum emission at a 0 . $^{\prime} 03$ resolution.
}

optically thin (Sect. 3.1). Figure 7 presents spectra of the $\mathrm{C}^{18} \mathrm{O}$ emission extracted from the combined data at four different positions along the blueshifted arc-like structure. The positions to extract spectra are labeled as A-D in Fig. 5b. At positions $\mathrm{A}-\mathrm{C}$, there are clearly two velocity components, one peaked at $V_{\mathrm{LSR}}$ of $\sim 6.5 \mathrm{~km} \mathrm{~s}^{-1}$ and the other peaked at higher velocities of $V_{\mathrm{LSR}}=4.5-5 \mathrm{~km} \mathrm{~s}^{-1}$. The component at the lower velocity is most likely associated with the largescale ambient gas, and the one at the higher velocities is the blueshifted arc-like structure. In Fig. 7, we also plotted the expected line-of-sight velocity from the best-matched model of a free-falling and rotating envelope in Yen et al. (2017) at these positions (blue vertical lines). This comparison shows that the relative velocities of the 


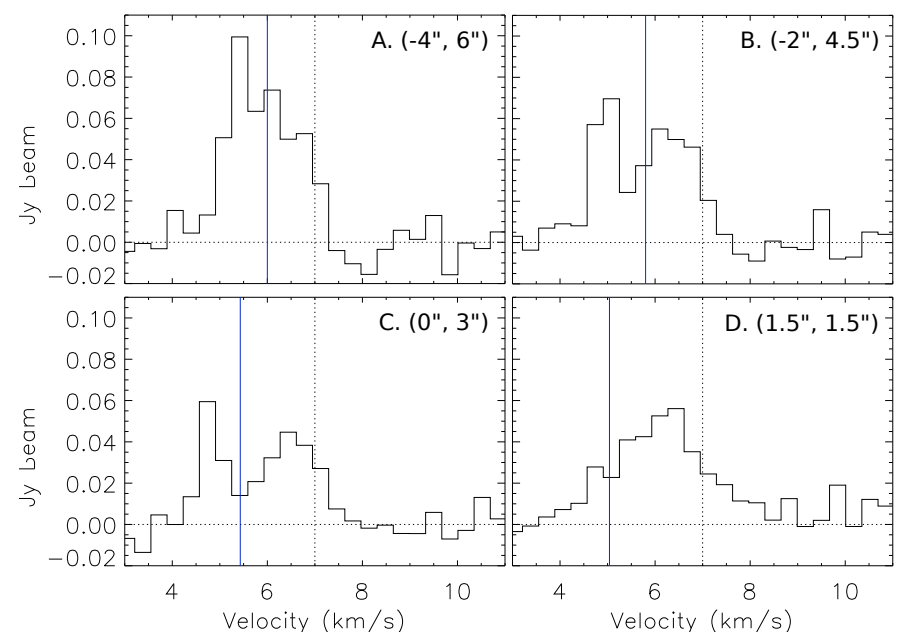

Fig. 7. Spectra of the $\mathrm{C}^{18} \mathrm{O}(2-1)$ emission extracted from the combined data. The relative offsets of the positions A-D, where the spectra were extracted, with respect to the position of HL Tau are shown at the upper right corners in the panels. These positions are also labeled in Fig. 5. Dotted vertical lines denote the systemic velocity of HL Tau, $V_{\mathrm{LSR}}=7 \mathrm{~km} \mathrm{~s}^{-1}$, measured from the Keplerian rotation of its disk (Yen et al. 2017). Blue vertical lines show the expected line-of-sight velocities from the model of a free-falling and rotating envelope at those positions (Yen et al. 2017).

blueshifted arc-like structure are indeed higher than the expected free-fall velocity, and the velocity excess is not due to the missing flux or the absorption.

\section{Analysis}

\subsection{Kinematics of the dense cloud around HL Tau}

HL Tau is located at the western edge of the shell-like structure with a size of $2^{\prime} \times 1.5(\sim 11000$ au in radius $)$ centered at XZ Tau observed in the ${ }^{13} \mathrm{CO}(1-0)$ emission (Welch et al. 2000). The position-velocity (PV) diagrams cutting through this shell show arc-like velocity profiles (Welch et al. 2000), which can be explained with an expanding shell (Arce et al. 2011; Offner \& Arce 2015). Thus, based on the intensity distribution and the velocity profiles of the ${ }^{13} \mathrm{CO}(1-0)$ emission, Welch et al. (2000) suggested that there is an expanding shell driven by XZ Tau, which is a T Tauri star and has launched wide-angle wind and molecular outflows (Krist et al. 1999, 2008; Zapata et al. 2015).

To examine this scenario of the largescale expanding shell in the HL Tau region with our IRAM $30 \mathrm{~m}$ data of the $\mathrm{C}^{18} \mathrm{O}(2-1)$ emission, which traces the density distribution of the cold dense gas and has less contamination from the outflow, we extracted a series of PV diagrams centered at the position of XZ Tau and along the position angles (PA) from $10^{\circ}$ to $170^{\circ}$ in steps of $20^{\circ}$. These PV diagrams indeed show arc-like velocity profiles, as expected for an expanding shell (Fig. 8). We note that there is no redshifted counterpart of the arc-like velocity profiles in these PV diagrams. For a spherical expanding shell, we expect to see both blue- and redshifted arc-like velocity profiles in PV diagrams (Arce et al. 2011). This suggests that the shell seen in the ${ }^{13} \mathrm{CO}(1-0)$ map in Welch et al. (2000) is not a spherical shell, and that XZ Tau is most likely located behind the dense cloud around HL Tau and the observed expanding shell. The dense cloud on the far side, behind XZ Tau, is possibly already blowed away by the wind and outflow from XZ Tau, and thus, no clear redshifted counterpart of the expanding shell is observed. This was also pointed out by Welch et al. (2000). In addition, the visual extinction $\left(A_{V}\right)$ of $\mathrm{XZ}$ Tau is measured to be 2.9 magnitude (Furlan et al. 2006). $A_{V}$ of 2.9 corresponds to a $\mathrm{H}_{2}$ column density of a few $\times 10^{21} \mathrm{~cm}^{-2}$ in the Taurus region on the assumption of the $\mathrm{CO}$ abundance of $10^{-4}$ (Pineda et al. 2010), suggesting that there is indeed cloud material in front of XZ Tau. In Fig. 9, we present a schematic figure of the proposed relative positions between XZ Tau, HL Tau, and the expanding shell.

To measure the velocity of the expanding motion, we compared the PV diagrams with the expected velocity profiles for an expanding shell. We assumed that the observed expanding motion is driven by wind or outflow from XZ Tau and is spherical symmetric with respect to the position of XZ Tau, and that the current radius of the expanding shell $\left(R_{\mathrm{sh}}\right)$ is $90^{\prime \prime}$, which is the radius of the shell observed in the ${ }^{13} \mathrm{CO}$ emission by Welch et al. (2000). In addition, we have assumed that the systemic velocity $\left(V_{\text {sys }}\right)$ of the largescale cloud around XZ Tau and HL Tau, meaning the cloud velocity before being swept up by wind or outflow from XZ Tau, is the same as $V_{\text {sys }}$ of HL Tau, $V_{\mathrm{LSR}}=7 \mathrm{~km} \mathrm{~s}^{-1}$. This $V_{\text {sys }}$ is also the centroid velocity of the $\mathrm{C}^{18} \mathrm{O}$ emission observed in the southwestern region, where is further away from XZ Tau (Fig. 2). Then, the line-of-sight velocity ( $\left.V_{\text {los }}\right)$ of the expanding motion at a given offset $\theta$ with respect to XZ Tau can be computed as

$\zeta=\arcsin \frac{\theta}{R_{\mathrm{sh}}}$,

and

$V_{\mathrm{los}}=V_{\mathrm{ex}} \cos \zeta+V_{\mathrm{sys}}$,

where $\zeta$ is the angle between the radial direction and the line of sight. A schematic figure of our model of the expanding shell is presented in Fig. 10.

With Eqs. (2) and (3), we computed the expected profiles for the expanding motion at three different velocities, $V_{\mathrm{ex}}=0.3,0.6$, and $0.9 \mathrm{~km} \mathrm{~s}^{-1}$. The observed velocity structures can be well described with the expected profiles for the expanding motion, especially in the $\mathrm{PV}$ diagrams of $\mathrm{PA}$ from $130^{\circ}$ to $10^{\circ}$, where the majority of the emission is all within the region enclosed by the expected profiles. In the PV diagrams of PA from $30^{\circ}$ to $90^{\circ}$, the velocity structures at the positive offsets can also be described with these expected profiles, while there is an intensity peak at the negative offset and at a velocity close to the assumed cloud velocity, $V_{\mathrm{LSR}}$ of $7 \mathrm{~km} \mathrm{~s}^{-1}$, which is offset from the expected profiles for the expanding motion. This component is located in the southwestern region in Fig. 1, which is brighter and further away from XZ Tau. Thus, this region may be less affected by the wind or outflow from XZ Tau and does not exhibit significant expanding motion. The position of HL Tau is at the offset of $-20^{\prime \prime}$ in the PV diagram of PA of $90^{\circ}$, where the majority of the emission is also within the velocity range enclosed by the expected profiles for the expanding motion, suggesting that the envelope around HL Tau could be also affected by the wind or outflow from XZ Tau.

In summary, the velocity structures observed in the $\mathrm{C}^{18} \mathrm{O}$ emission suggest that the largescale ambient gas around HL Tau is likely expanding at velocities of $0.3-0.9 \mathrm{~km} \mathrm{~s}^{-1}$. The estimated $V_{\text {ex }}$ is comparable to or lower than that in the ${ }^{13} \mathrm{CO}$ emission by Welch et al. (2000), $1.2 \mathrm{~km} \mathrm{~s}^{-1}$. This difference could be due to different emission lines adopted in the analyses, and our $\mathrm{C}^{18} \mathrm{O}(2-1)$ observations are more sensitive to denser gas compared to the ${ }^{13} \mathrm{CO}$ observations. 

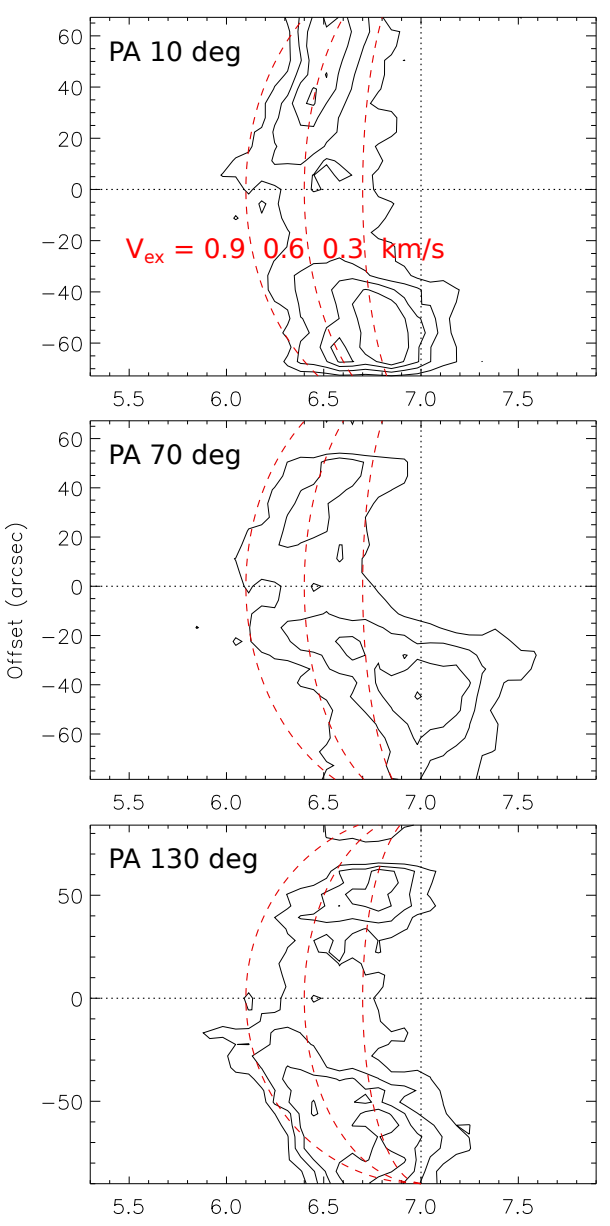
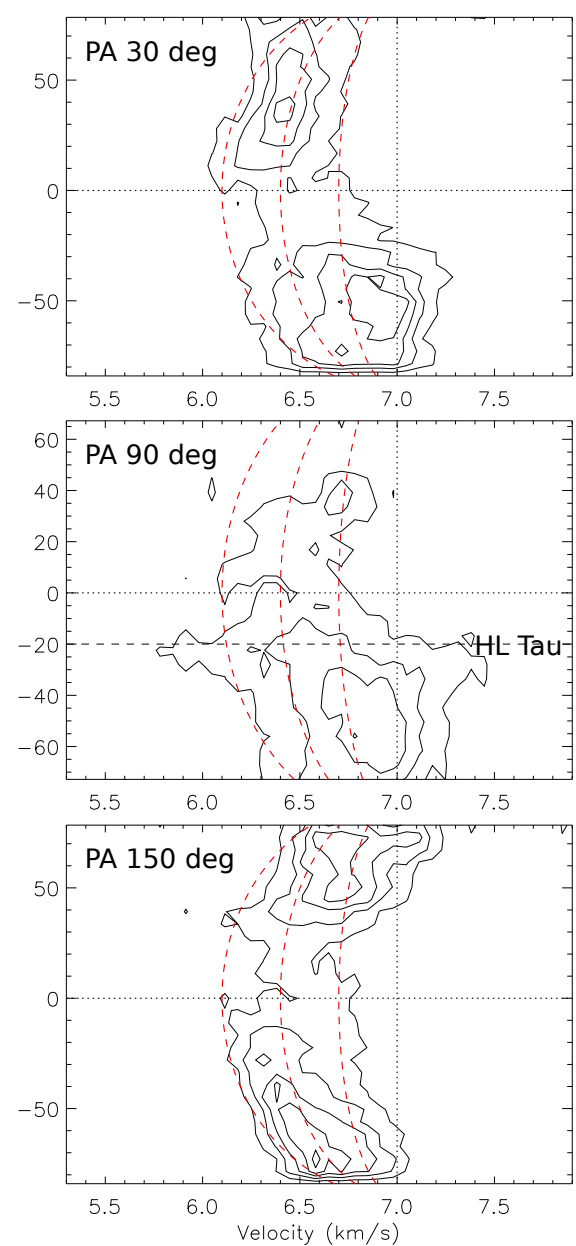
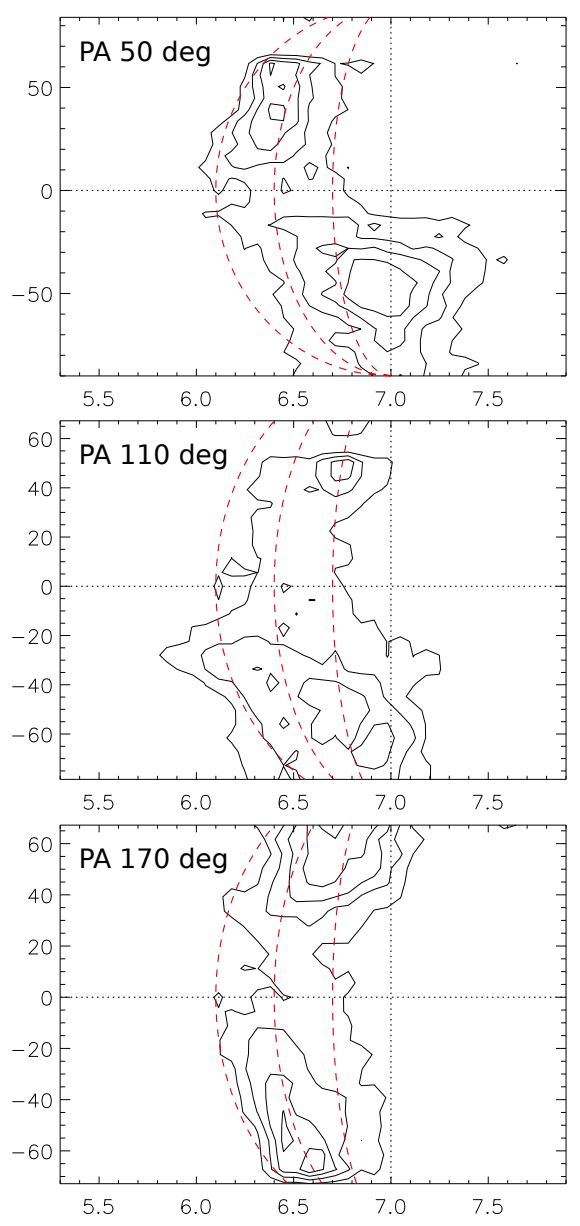

Fig. 8. Position-velocity (PV) diagrams of the $\mathrm{C}^{18} \mathrm{O}(2-1)$ emission obtained with the IRAM 30m observations. The PV diagrams were extracted along different position angles (labeled at the upper left corner in each panel) and passing through the position of XZ Tau, which is shown as zero offset and denoted with horizontal dotted lines. Vertical dotted lines present the systemic velocity of HL Tau measured from the Keplerian rotation of the HL Tau disk. The position of HL Tau is at the offset of $-20^{\prime \prime}$, denoted as a horizontal dashed line, in the PV diagram along PA of $90^{\circ}$. Red dashed curves from right to left in each panel present the expected velocity profiles of the expanding shell with expanding velocities of 0.3 , 0.6 , and $0.9 \mathrm{~km} \mathrm{~s}^{-1}$, respectively, as labeled in the PV diagram along PA of $10^{\circ}$.

\subsection{Kinematical model of the arc structure in HL Tau}

Our combined maps of the $\mathrm{C}^{18} \mathrm{O}$ emission show that the observed velocity in the blueshifted arc-like structure connecting to the disk around HL Tau is more blueshifted than the expected line-of-sight velocity from the free-fall motion (Fig. 7), and this blueshifted velocity excess is not due to the effects of missing flux or optical depth. In addition, our single-dish ${ }^{13} \mathrm{CO}$ and $\mathrm{C}^{18} \mathrm{O}$ spectra at the position of HL Tau also shows that the velocities of the intensity peaks are $0.3-0.6 \mathrm{~km} \mathrm{~s}^{-1}$ more blueshifted than the systemic velocity of HL Tau (Fig. 2). These blueshifted velocity excesses in the arc-like structure and the protostellar envelope could be naturally explained if the protostellar envelope around HL Tau has a relative motion toward us with respect to HL Tau.

To examine this possibility, we constructed a kinematical model of an infalling and rotating protostellar envelope including a relative motion between the envelope and the central protostar. Because of this relative motion, the infalling and rotational motions in the model envelope cannot be simply described with any conventional models, such as that in Ulrich (1976). Thus, we constructed a new kinematical model by placing a set of test particles and calculating their trajectories and velocities with time evolution based on the equations of motion.
Then, we projected their positions on the plane of the sky and their velocities on the line of sight to compare with our observations.

We first assigned each particle an initial velocity computed with the conventional model of an infalling and rotating envelope by Ulrich (1976). In our model, the mass of the central star is adopted to be $1.8 M_{\odot}$, the same as HL Tau (Yen et al. 2017), and the centrifugal radius is adopted to be $100 \mathrm{au}$, the observed radius of the disk around HL Tau (ALMA Partnership 2015). The inclination and position angles of the model envelope are also adopted to be the same as the disk around HL Tau, $47^{\circ}$ and $138^{\circ}$, respectively (ALMA Partnership 2015). Then, to introduce a relative motion between the model envelope and the central star, we added an additional velocity component $\left(V_{\text {rel }}\right)$ to all the particles in addition to their initial infalling and rotational velocities. The direction of this additional velocity is along the line of sight and toward us. As a summary of this scenario, HL Tau first formed out of a protostellar envelope with $V_{\text {sys }}$ of $7 \mathrm{~km} \mathrm{~s}^{-1}$, which is the $V_{\text {sys }}$ of the disk around HL Tau. Then after HL Tau has accumulated its current stellar mass and the 100 au disk has formed, the protostellar envelope starts to move relatively with respect to HL Tau. Our kinematical model starts with the protostellar mass of $1.8 M_{\odot}$ and the disk radius of $100 \mathrm{au}$, and the 


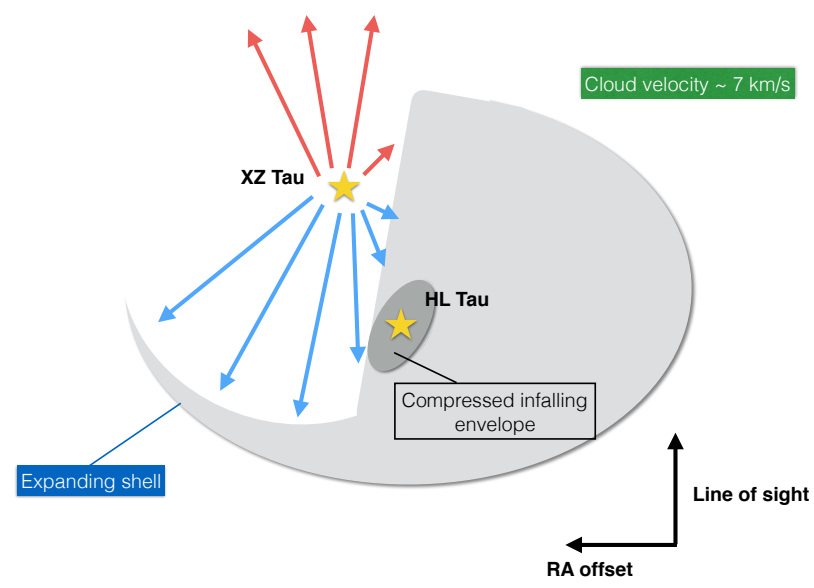

Fig. 9. Schematic figure of the proposed relative positions between HL Tau, XZ Tau, and the expanding shell. Horizontal and vertical directions are the directions of RA offset and the line of sight, respectively.

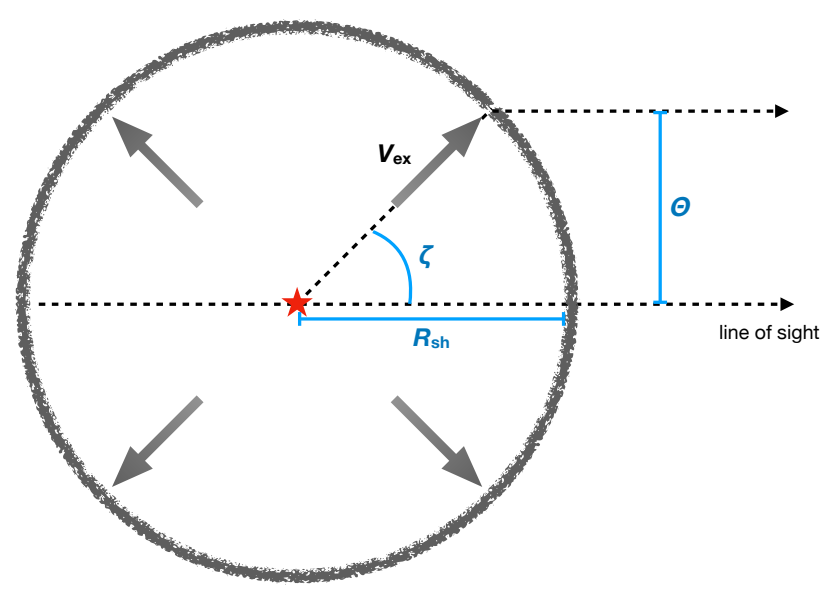

Fig. 10. Schematic figure of our model of the expanding shell (gray open circle) to illustrate Eqs. (2) and (3). The expansion (gray arrows) is assumed to be spherical and centered at the position of XZ Tau, labelled as a red star. $R_{\mathrm{sh}}$ is the radius of the expanding shell. $\theta$ is the positional offset with respect to XZ Tau on the plane of the sky. $\zeta$ is the angle between the radial direction and the line of sight.

relative motion between the envelope and the protostar is added from the start of our model.

We first started with axisymmetric envelope models. Test particles were distributed within a radius of 3000 au and $40^{\circ}$ from the midplane to mimic the flattened envelope as observed in HL Tau (Hayashi et al. 1993). The number density of the test particles in the model envelope is proportional to $r^{-1.5}$, the same as the conventional expectation for an infalling envelope (Shu 1977). With the initial setup of the particle distribution and the velocity field, we then computed the evolution of the model envelopes having different $V_{\text {rel }}$ of $0,0.3,0.6$, and $0.9 \mathrm{~km} \mathrm{~s}^{-1}$. Considering that the envelope mass in HL Tau is only 0.03 $0.06 M_{\odot}$ on a scale of 20" (2800 au; Hayashi et al. 1993; Cabrit et al. 1996) and is $0.13 M_{\odot}$ within a radius of 4200 au (Motte \& André 2001), less than $10 \%$ of the stellar mass of HL Tau, the self gravity of the model envelope can be safely ignored. In our calculations, the gravity of the central star is assumed to be the only source of the acceleration in the equations of motion, and all the particles are assumed to be collisionless. In addition, we assume that the particles are accreted onto the disk if they enter the disk region, which is assumed to be a cylinder with a radius of $100 \mathrm{au}$ and a constant height of $10 \mathrm{au}$. These accreted particles were removed from the calculations. We let these model envelopes evolve for $10^{4} \mathrm{yr}$, which is approximately the dynamical timescale for a particle at the outer radius of our model envelope to fall into the center at its free-fall velocity. As discussed below, this evolutionary time is an arbitrary choice and is not a unique solution to explain the observational results.

After computing the evolution of the model envelopes, we generated synthetic images in the $\mathrm{C}^{18} \mathrm{O}$ emission of our model envelope. To compute synthetic image cubes of the $\mathrm{C}^{18} \mathrm{O}$ emission, for a given position, the number density and motional velocity of $\mathrm{C}^{18} \mathrm{O}$ are adopted to be the number density and mean velocity of the test particles at that position in our kinematical models. The temperature in our model envelope is adopted from interpolation of the observational estimates. The temperature at the disk outer radius of $100 \mathrm{au}$ in HL Tau is measured to be $60 \mathrm{~K}$ from the $\mathrm{CO}(1-0)$ brightness temperature observed with ALMA (Yen et al. 2016), on the assumption that the CO (1-0) line is optically thick and its brightness temperature is the kinematic temperature. On the other hand, the temperature at a radius of 1000 au in the blueshifted arc-like structure in HL Tau is estimated to be $15 \mathrm{~K}$ from the intensity ratio of the ${ }^{13} \mathrm{CO}(2-1)$ and $\mathrm{C}^{18} \mathrm{O}(2-1)$ lines (Yen et al. 2017), on the assumption of a ${ }^{13} \mathrm{CO} / \mathrm{C}^{18} \mathrm{O}$ abundance ratio of 10 (Brittain et al. 2005; Smith et al. 2015). We assumed that the radial profile of the temperature in the envelope is a power-law function. By interpolating these two temperature measurements at radii of 60 and $1000 \mathrm{au}$, the temperature profile in our model envelope is adopted to be $T(r)=15 \times(r / 1000 \mathrm{au})^{-0.6} \mathrm{~K}$. Then, the $\mathrm{C}^{18} \mathrm{O}$ intensity in the synthetic images was computed with the radiative transfer equation and integrated along the line of sight, and we scaled the total number of $\mathrm{C}^{18} \mathrm{O}$ in the model to make the $\mathrm{C}^{18} \mathrm{O}$ intensity in our synthetic images comparable to that in the observations. Finally, the synthetic images were convolved with the same synthesized beam as the observations. We note that there is no disk component in our synthetic images because our kinematical models do not include a disk.

We note that none of these axisymmetric envelope models form arc-like structures in their synthetic velocity channel maps of the $\mathrm{C}^{18} \mathrm{O}$ emission. We find that arc-like structures similar to the observations form in the synthetic velocity channel maps when the initial particle distributions of our kinematical models are not axisymmetric. To derive the initial particle distributions in the kinematical models that could result in arc-like structures in the synthetic images, we compared the observed velocity channel maps and the evolved particle distributions from our kinematical models projected on the plane of the sky at the same line-of-sight velocities. We identified those particles whose positions do not overlap with the observed intensity distribution of the $\mathrm{C}^{18} \mathrm{O}$ emission, and removed them from the initial particle distributions in our kinematical models. An example to derive the initial particle distribution with a given $V_{\text {rel }}$ and an evolutionary time is presented in Appendix A. The derived initial particle distributions for the kinematical models with the adopted $V_{\text {rel }}$ of $0,0.3,0.6$, and $0.9 \mathrm{~km} \mathrm{~s}^{-1}$ and evolutionary time of $10^{4} \mathrm{yr}$ projected on the plane of the sky are shown in Fig. 11. Because of the trajectories and velocities of the particle motions in our kinematical models with different $V_{\text {rel }}$ are different, the derived initial particle distributions for different $V_{\text {rel }}$ are different. In addition, when a different evolutionary time is adopted, the derived initial 

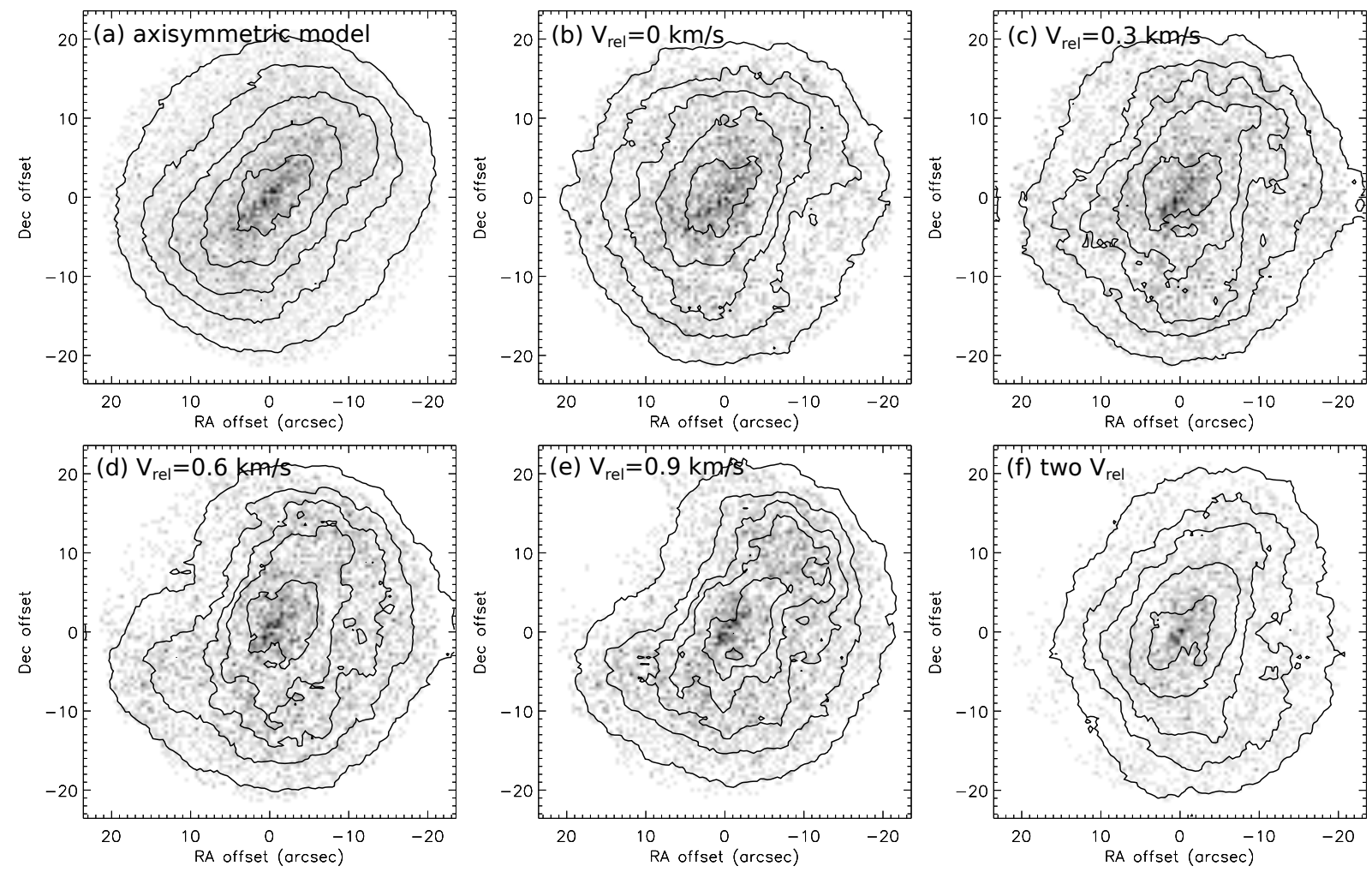

Fig. 11. Initial particle distributions (grayscale) in our kinematical models projected on the plane of the sky, which represent the maps of the column density of our model envelopes. Panel $a$ : case of the axisymmetric envelope model. Panels $b-e$ : cases of the asymmetric envelope models with different $V_{\text {rel }}$ as labeled at the upper left corners. Panel $f$ : model of the asymmetric envelope with the two $V_{\text {rel }}, V_{\text {rel }}$ of 0.9 and $0 \mathrm{~km} \mathrm{~s}{ }^{-1}$ on the far and near sides of the envelope, respectively. Contours show the fraction of the total number of the particles, starting from $10 \%$ in steps of $20 \%$.

particle distribution is also different. With our model approach, an initial particle distribution, which can form arc-like structures after the evolution, can be found for a given set of $V_{\text {rel }}$ and an evolutionary time. Thus, there is no unique combination of $V_{\text {rel }}$ and an evolutionary time to explain the observation.

Figure 11 shows that when there is less envelope material in the northeastern region (for models with higher $V_{\text {rel }}$ ) and the southwestern region (for models with lower $V_{\text {vel }}$ ), the model envelope could form arc-like structures in its synthetic velocity channel maps after it evolves. This distribution with less envelope material in the northeastern and southwestern regions is similar to the observed gas distribution on a scale of thousands of au around HL Tau, which shows an elongation from the northwest to the south of HL Tau (Fig. 1 and Welch et al. 2000). With the derived initial particle distributions in Fig. 11, we computed our kinematical models again and generated synthetic images (Figs. 12 and 13).

Our synthetic images of the model with $V_{\text {vel }}$ of $0 \mathrm{~km} \mathrm{~s}^{-1}$ can well explain the observed intensity distribution at the redshifted velocity of $V_{\mathrm{LSR}}$ of $7.5-9.2 \mathrm{~km} \mathrm{~s}^{-1}$, but the blueshifted emission at $V_{\mathrm{LSR}}$ of $4.7-5.4 \mathrm{~km} \mathrm{~s}^{-1}$ in the synthetic images is less extended than the observations (Fig. 12). Especially, in the synthetic images with $V_{\text {vel }}$ of $0 \mathrm{~km} \mathrm{~s}^{-1}$, there is no counterpart of the northwestern peak in the blueshifted arc-like structure observed at $V_{\mathrm{LSR}}$ of $4.7-5.4 \mathrm{~km} \mathrm{~s}^{-1}$. In contrast, the synthetic images of the model with $V_{\text {vel }}$ of $0.6 \mathrm{~km} \mathrm{~s}^{-1}$ can explain the morphology and velocity of the observed blueshifted arc-like structure at $V_{\mathrm{LSR}}$ of $4.7-5.4 \mathrm{~km} \mathrm{~s}^{-1}$, but the intensity distribution in the synthetic images at the redshifted velocity of $V_{\mathrm{LSR}}$ of $7.5-8.1 \mathrm{~km} \mathrm{~s}^{-1}$ is less extended than the observations (Fig. 13). The synthetic images of the kinematical models with $V_{\mathrm{vel}}$ of 0.3 and $0.9 \mathrm{~km} \mathrm{~s}^{-1}$ are shown in Appendix B. The comparison of the observed velocity channel maps with these four models shows that the observed blueshifted emission can be better explained with the models with higher $V_{\text {vel }}$ of 0.6 and $0.9 \mathrm{~km} \mathrm{~s}^{-1}$, while the observed redshifted emission with the models with no or lower $V_{\text {vel }}$ of $0.3 \mathrm{~km} \mathrm{~s}^{-1}$. We also tested other combinations of evolutionary time and initial density distributions. We find that our kinematical models with $V_{\text {rel }}$ of 0.6 and $0.9 \mathrm{~km} \mathrm{~s}^{-1}$ still form arc-like structures at the blueshifted velocity similar to the observations in the synthetic velocity channel maps, and that $V_{\text {rel }}$ of $0.6-0.9 \mathrm{~km} \mathrm{~s}^{-1}$ is needed in our kinematical models to explain the high velocity observed in the blueshifted arc-like structure.

In HL Tau, the $\mathrm{C}^{18} \mathrm{O}$ emission at blue- and redshifted velocities traces the far and near sides of the infalling protostellar envelope, respectively (Yen et al. 2017). Thus, we constructed an additional model with $V_{\text {rel }}$ of $0.9 \mathrm{~km} \mathrm{~s}^{-1}$ on the far side and $V_{\text {rel }}$ of $0 \mathrm{~km} \mathrm{~s}^{-1}$ on the near side of the envelope, and generated synthetic images. The initial particle distribution of this model with the two $V_{\text {rel }}$ is shown in Fig. 11f, and its synthetic images in Fig. 14. Its initial particle distribution is elongated from the northwest to the south, similar to the observed intensity distributions of the $\mathrm{C}^{18} \mathrm{O}$ lines with the single-dish telescopes (Fig. 1). The synthetic images from this kinematical model can well explain the channel maps at both the blue- and redshifted velocities. We note that the model with the two $V_{\text {rel }}$ has less emission at $V_{\mathrm{LSR}}$ of 6.4 and $6.8 \mathrm{~km} \mathrm{~s}^{-1}$ compared to the observations. This could be due to the largescale ambient gas with the peak velocity of $6.7 \mathrm{~km} \mathrm{~s}^{-1}$ around the protostellar envelope (Figs. 1 and 2) not included in our model. 


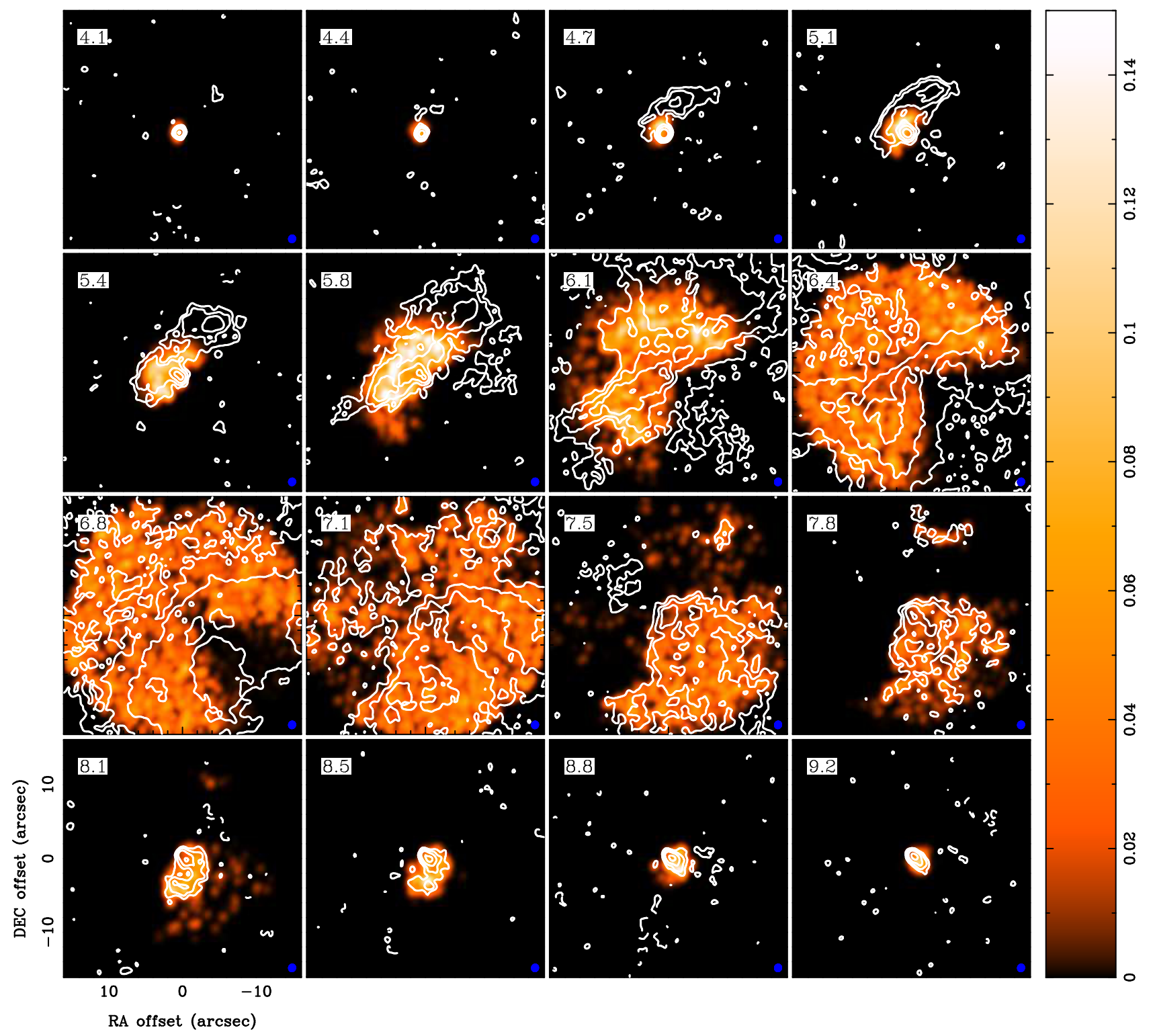

Fig. 12. Observed (contour) and synthetic (colorscale in units of $\mathrm{Jy} \mathrm{Beam}^{-1}$ ) velocity channel maps of the $\mathrm{C}^{18} \mathrm{O}$ emission. The synthetic maps were generated from the kinematical model with $V_{\text {rel }}$ of $0 \mathrm{~km} \mathrm{~s}^{-1}$ and the asymmetric initial density distribution presented in Fig. 11. Contour levels are from $3 \sigma$ and increase in steps of a factor of two, where $1 \sigma$ is $5.5 \mathrm{mJy} \mathrm{Beam}^{-1}$. The central velocity of each channel is labeled at the upper left corner in each panel in units of $\mathrm{km} \mathrm{s}^{-1}$. Blue filled ellipses show the size of the synthesized beam.

\section{Discussion}

\subsection{Possible relative motion between HL Tau and its protostellar envelope}

The observed velocities and morphologies of the arc-like structures connected to the disk around HL Tau cannot be explained with any conventional model of an infalling and rotating envelope, and the observed velocity in the blueshifted arc-like structure is higher than the expectation from the free-fall motion. With our new kinematical models, the high velocity observed in the blueshifted arc-like structure connecting to the disk can be explained by including a relative motion between HL Tau and its protostellar envelope in addition to the original infalling and rotational motions. The morphologies of the arc-like structures can also be reproduced with our kinematical models with asymmetric initial density distributions (Fig. 11). In addition, the comparison between the observations and our kinematical models with the relative motions at different velocities suggests that the far side of the envelope has a higher additional relative velocity with respect to HL Tau, while the near side of the envelope has a lower or no additional relative velocity. Our kinematical models show that when the far side of the model envelope has an additional relative velocity of $0.6-0.9 \mathrm{~km} \mathrm{~s}^{-1}$ along the line of sight with respect to the central protostar, the observed velocity in the blueshifted arc-like structure can be well reproduced.

There are two possibilities in which the protostellar envelope moves relatively with respect to HL Tau: the envelope suffered an external impact or HL Tau is attracted by other nearby objects. Our single-dish results support the presence of an expanding shell driven by XZ Tau, as suggested by Welch et al. (2000). The expanding velocity is estimated to be $0.3-0.9 \mathrm{~km} \mathrm{~s}^{-1}$ with our $\mathrm{C}^{18} \mathrm{O}(2-1)$ observations and to be $1.2 \mathrm{~km} \mathrm{~s}^{-1}$ with the ${ }^{13} \mathrm{CO}(1-0)$ observations by Welch et al. (2000). We note that 


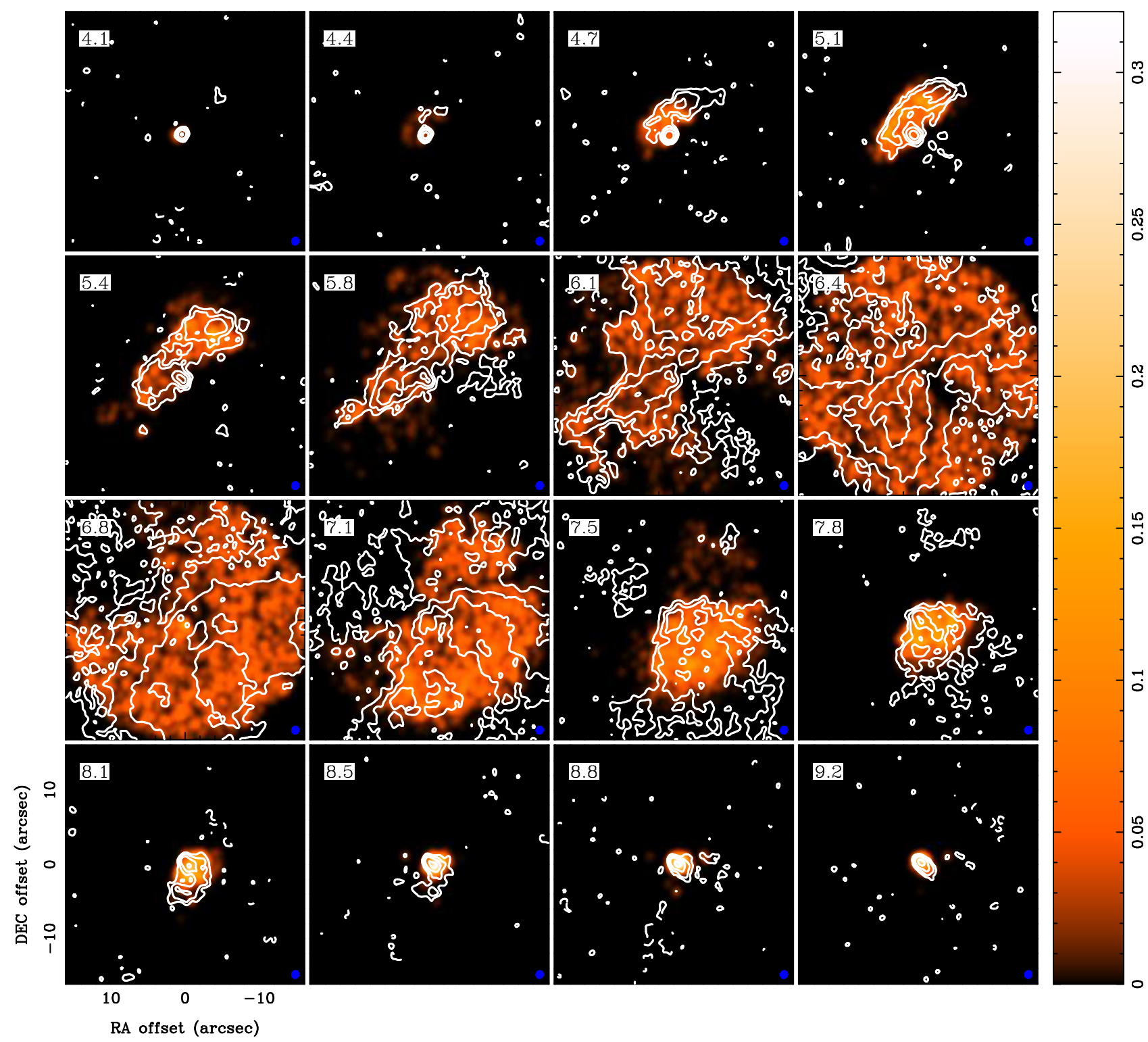

Fig. 13. Same as Fig. 12 but with $V_{\text {rel }}$ of $0.6 \mathrm{~km} \mathrm{~s}^{-1}$.

the velocity of the wind or outflow driving this largescale expansion can be much higher than this expanding velocity (Welch et al. 2000). HL Tau is located at the edge of this expanding shell, and our single-dish ${ }^{13} \mathrm{CO}$ and $\mathrm{C}^{18} \mathrm{O}$ spectra show that the ambient gas on a 1000 au scale around HL Tau is strongly blueshifted with respect to the systemic velocity of HL Tau by $0.3-0.6 \mathrm{~km} \mathrm{~s}^{-1}$. These results suggest that the protostellar envelope around HL Tau can be impacted by the largescale expanding motion (Sect. 4.1), which was also suggested by Welch et al. (2000). The estimated expanding velocity is comparable to the required velocity of the relative motion between HL Tau and its protostellar envelope to explain the observed velocity structures in the combined maps with our kinematical models. In addition, our kinematical models suggest that the relative velocity between HL Tau and its protostellar envelope is higher on the far side and lower on the near side of the envelope. The candidate driving source of the largescale expanding motion, XZ Tau, is most likely located behind HL Tau along the direction of the line of sight (Fig. 9). Thus, the far side of the envelope, which is closer to the driving source, likely gains more momentum from the largescale expanding motion and has a higher additional relative velocity, while the near side is shielded by the far side and is less impacted by the expanding motion. Therefore, the observed velocity structures and morphology of the protostellar envelope are consistent with the expectation from this scenario of the impact by the largescale expanding motion driven by wind or outflow from the T Tauri star XZ Tau.

In this scenario, the three-dimensional distance between HL Tau and the driving source of the largescale expansion, $\mathrm{XZ} \mathrm{Tau}$, is $90^{\prime \prime}$ (12600 au) based on the observed radius of the expanding shell (Fig. 10). If the expanding shell originates from the position of XZ Tau with a constant expanding velocity of $1 \mathrm{~km} \mathrm{~s}^{-1}$, it takes $5 \times 10^{4} \mathrm{yr}$ for the expanding shell to reach the position of HL Tau. On the other hand, the expansion is likely driven by the wind or outflow from XZ Tau whose velocity is much higher than the expanding velocity of the shell. With a wind or outflow velocity of $10 \mathrm{~km} \mathrm{~s}^{-1}$, the wind or outflow reaches the position of HL Tau $5 \times 10^{3}$ yr after its launch. Depending on the initial density distribution of the ambient cloud around HL Tau and XZ Tau, it is most likely that the 


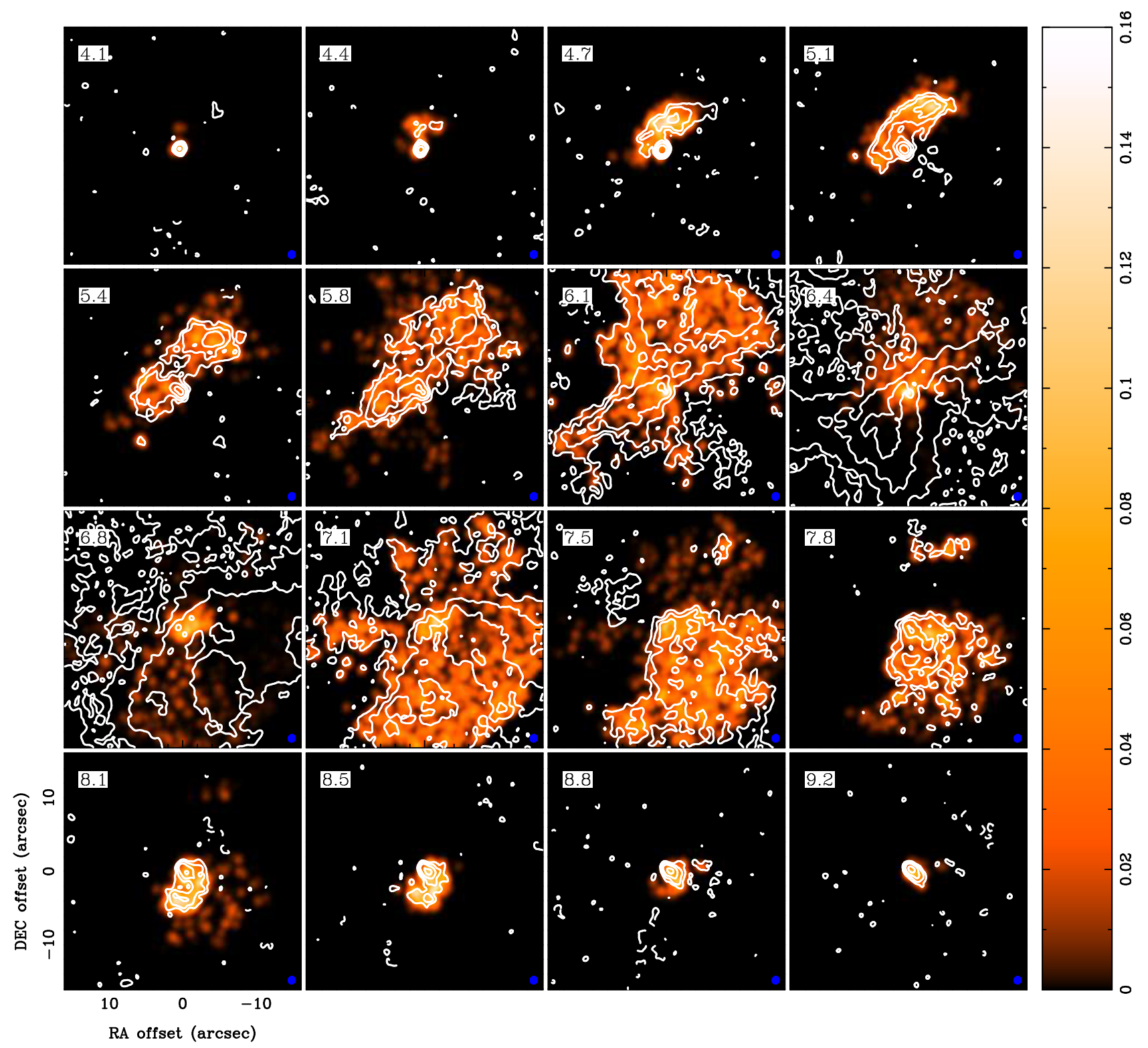

Fig. 14. Same as Fig. 12 but with the two $V_{\text {rel }}, 0.9 \mathrm{~km} \mathrm{~s}^{-1}$ on the far side and $0 \mathrm{~km} \mathrm{~s}^{-1}$ on the near side of the envelope.

wind or outflow from XZ Tau first travels at a high velocity of tens of $\mathrm{km} \mathrm{s}^{-1}$ and impacts the ambient cloud to form the expanding shell, and then the shell expands at a low velocity of $0.6-1.2 \mathrm{~km} \mathrm{~s}^{-1}$, reaches and impacts the envelope around HL Tau. Therefore, the impact on the envelope around HL Tau by the largescale expansion possibly occurred between $5 \times 10^{3}$ and $5 \times 10^{4}$ ago. In our kinematical model, we adopt an evolutionary time of $10^{4} \mathrm{yr}$, and it is not a unique timescale to explain the observational results. Thus, considering the timescales of the propagation of the wind or outflow from XZ Tau and the largescale expansion, the scenario of the external impact is indeed possible.

Alternatively, the relative motion between HL Tau and its protosteller envelope could be due to HL Tau being attracted by nearby objects, such as XZ Tau, and moving relatively to its envelope. However, this scenario cannot explain why the protostellar envelope is not also being attracted by the same gravitational source and moves together with HL Tau. The protostellar envelope around HL Tau is embedded in the filamentary structure, and there is not much gas around XZ Tau (Fig. 1). The pressure gradient of such a gas distribution is unlikely to support the protostellar envelope not to be attracted and move together with HL Tau, if there is indeed a gravitational source to drag HL Tau. Therefore, this scenario of HL Tau being attracted and leaving its protostellar envelope is unlikely.

\subsection{Impact on the kinematics of protostellar envelopes by nearby outflow feedback}

Our results hint that the protostellar envelope around HL Tau is impacted by the largescale expanding motion driven by wind or outflow from the nearby young stellar object. Such an impact can affect the gas kinematics and thus evolution of the protostellar envelope around HL Tau. We have computed our kinematical models with different relative velocities between HL Tau and its envelope for an evolutionary time longer than $10^{4} \mathrm{yr}$. In the model with $V_{\text {rel }}$ of $0 \mathrm{~km} \mathrm{~s}^{-1}$, the entire envelope is eventually accreted onto the disk, meaning that all the test particles enter 
the disk region, within a timescale of $2 \times 10^{4} \mathrm{yr}$, as expected in the conventional model of an infalling and rotating envelope (e.g., Ulrich 1976). On the other hand, in the model envelope with $V_{\text {rel }}$ of $0.3,0.6$, and $0.9 \mathrm{~km} \mathrm{~s}^{-1}$, there are 60,80 , and $90 \%$ of particles, respectively, which do not encounter the disk region and leave the system. Because of the additional relative velocity, these particles become gravitationally unbounded, and their centrifugal radii become larger than the disk radius of $100 \mathrm{au}$. As a result, they eventually pass by the disk when they infall toward the center.

Thus, after the protostellar envelope around HL Tau is impacted by the largescale expanding motion, if there is no other mechanism, such as magnetic braking, to transfer the angular momenta of the envelope material away, a part of the envelope is unlikely to be accreted onto the disk around HL Tau. On the other hand, magnetic braking is expected to be inefficient in the later evolutionary stage when protostellar envelopes start to dissipate (e.g., Machida et al. 2011). In our kinematical models with the two $V_{\text {rel }}$, which can well explain the observed velocity channel maps at both the blue- and redshifted velocities, only the half of the model envelope is accreted onto the disk. Therefore, the mass infalling rate from the envelope onto the disk in HL Tau likely decreases by a factor of two due to the impact by the largescale expanding motion.

A similar impact of outflow launched by nearby protostars on protostellar envelopes has also been observed in the Class I protostar, L1551 NE. The protostellar envelope around L1551 NE shows the signatures of dissipating motion, which is likely caused by the impact of the outflow from the nearby protostar, L1551 IRS5 (Takakuwa et al. 2015). It is expected that there is no further mass infall onto the circumbinary disk around L1551 NE (Takakuwa et al. 2015). L1448C(S) is also a candidate protostar having such impact of outflow on its protostellar envelope (Hirano et al. 2010). In the L1448 region, the position of $\mathrm{L} 1448 \mathrm{C}(\mathrm{S})$ on the plane of the sky overlaps with the outflow launched by L1448C(N). L1448C(S) is surrounded by a small amount of circumstellar material $\left(<0.01 M_{\odot}\right)$ traced by the $860 \mu \mathrm{m}$ continuum and less obscured in infrared, compared to L1448C(N), suggesting that the envelope around L1448C(S) can be stripped away by the outflow of L1448C(N) (Hirano et al. 2010).

Such influence of the outflow feedback on protostellar sources may not be rare. Expanding shells similar to that around XZ Tau have often been observed in star-forming regions. With Nobeyama $45 \mathrm{~m}$ observations in the CO $(1-0)$ and ${ }^{13} \mathrm{CO}(1-0)$ emission, 42 expanding shells were identified in the massive star-forming region, Orion $\mathrm{A}$, and the radii and expanding velocities of these shells range from 0.05 to $0.85 \mathrm{pc}$ and from 0.8 to $5 \mathrm{~km} \mathrm{~s}^{-1}$, repsectively (Feddersen et al. 2018). Many expanding shells have also been observed in low- or intermediate-mass starforming regions. In the Perseus star-forming region, 12 expanding shells with radii of $0.1-3 \mathrm{pc}$ and expanding velocities of $1-6 \mathrm{~km} \mathrm{~s}^{-1}$ were identified with the COMPLETE survey in the CO (1-0) and ${ }^{13} \mathrm{CO}(1-0)$ lines (Arce et al. 2011). In the Taurus star-forming region, 37 expanding shells were found in the FCRAO observations in the $\mathrm{CO}(1-0)$ and ${ }^{13} \mathrm{CO}(1-0)$ lines (Li et al. 2015). These expanding shell are suggested to be driven by young stellar objects (Arce et al. 2011; Li et al. 2015; Feddersen et al. 2018).

The interaction between protostellar envelopes and associated outflows in protostellar sources has been suggested to affect the structures of the envelopes and to limit the volume of the infalling region, and eventually the protostellar envelopes dissipate and the mass infalling rates decrease
(Takakuwa et al. 2003; Arce \& Sargent 2006; Machida \& Matsumoto 2012). Our results suggest an additional path to decrease mass infalling rates in protostellar envelopes by outflow feedback from nearby young stellar objects.

\section{Summary}

We have conducted observations toward HL Tau in the ${ }^{13} \mathrm{CO}(3-2)$ and $\mathrm{C}^{18} \mathrm{O}(3-2)$ lines with JCMT and in the ${ }^{13} \mathrm{CO}(2-1)$ and $\mathrm{C}^{18} \mathrm{O}(2-1)$ lines with the IRAM 30m telescope and the ACA 7-m array, and generated combined images with the IRAM 30m, ACA, and ALMA data. With the single-dish and interferometric data, we have studied the gas motions on a $0.1 \mathrm{pc}$ scale in the HL Tau region and in the protostellar envelope on a $1000 \mathrm{au}$ scale around HL Tau. Our main results are summarized below.

- Our single-dish images of the $\mathrm{C}^{18} \mathrm{O}(3-2)$ and (2-1) emission show that HL Tau is located in a largescale $(0.1 \mathrm{pc})$ filamentary structure elongated from the northwest to the south of HL Tau. On the contrary, the ${ }^{13} \mathrm{CO}(3-2)$ and (2-1) emission is centrally peaked at the position of HL Tau and does not exhibit clear elongation. The ${ }^{13} \mathrm{CO}$ intensity distribution most likely has contribution from the outflow associated with HL Tau, while the $\mathrm{C}^{18} \mathrm{O}$ emission is less contaminated by the outflow and traces the structures of the largescale molecular cloud around HL Tau. The comparison of the intensity ratios between (3-2) and (2-1) transitions of the $\mathrm{C}^{18} \mathrm{O}$ and ${ }^{13} \mathrm{CO}$ lines shows that the $\mathrm{C}^{18} \mathrm{O}$ emission is optically thin, while the ${ }^{13} \mathrm{CO}$ emission is optically thick at the peak velocity $\left(V_{\mathrm{LSR}}=6.4 \mathrm{~km} \mathrm{~s}^{-1}\right)$ and is optically thin at the line wing $\left(V_{\mathrm{LSR}}<5.6 \mathrm{~km} \mathrm{~s}^{-1}\right.$ and $\left.>7.2 \mathrm{~km} \mathrm{~s}^{-1}\right)$.

- Our combined images generated from the IRAM 30m, ACA, and ALMA data show that the $\mathrm{C}^{18} \mathrm{O}(2-1)$ and ${ }^{13} \mathrm{CO}(2-1)$ emission lines at the high velocities, $\Delta V \lesssim-3 \mathrm{~km} \mathrm{~s}^{-1}$ and $\gtrsim 1.5 \mathrm{~km} \mathrm{~s}^{-1}$ with respect to the systemic velocity of HL Tau, primarily trace the rotation of the circumstellar disk on a 100 au scale around HL Tau. Blue- and redshifted arc-like structures are observed at the medium velocities, $|\Delta V|>0.8-1.4 \mathrm{~km} \mathrm{~s}^{-1}$. The sizes of the arc-like structures gradually increase with decreasing relative velocity, and the arc-like structures emerge with the largescale molecular cloud at the low velocity, $|\Delta V|<0.8-1.4 \mathrm{~km} \mathrm{~s}^{-1}$, suggesting that the arc-like structures are most likely formed by infalling material from the ambient gas around HL Tau. In addition, the $\mathrm{C}^{18} \mathrm{O}(2-1)$ spectra of the blueshifted arc-like structure from the combined data show that its relative velocity with respect to the systemic velocity of HL Tau is higher than the expected free-fall velocity, and this velocity excess is not due to the effects of missing flux or absorption.

- Position-velocity diagrams of the $\mathrm{C}^{18} \mathrm{O}(2-1)$ emission passing through the position of XZ Tau along several different position angles obtained with the IRAM 30m observations show arc-like velocity profiles at the blueshifted velocities, suggesting that the largescale molecular cloud in the HL Tau region has expanding motion, possibly driven by the wind or outflow from XZ Tau, as suggested by Welch et al. (2000). The velocity of the expanding motion is estimated to be $0.3-0.9 \mathrm{~km} \mathrm{~s}^{-1}$. HL Tau is located at the edge of this expanding shell. No signature of the expanding motion was observed at the redshifted velocity, suggesting that XZ Tau is located behind HL Tau along the line of sight, and the gas on the rear side of XZ Tau may already be blowed away.

- We constructed kinematical models of an infalling and rotating envelope including a relative motion between the 
central star and the envelope at velocities of $0,0.3,0.6$, and $0.9 \mathrm{~km} \mathrm{~s}^{-1}$. We computed the time evolution of the model envelope and generated synthetic images in the $\mathrm{C}^{18} \mathrm{O}(2-1)$ emission. We find that when the relative motion is included, our kinematical models can explain the observed high velocity in the blueshifted arc-like structure, and the morphologies of the observed arc-like structures can also be well reproduced with our models having an asymmetric initial density distribution. The comparison between our models and the observations suggests that in addition to the infalling and rotational motions in the protostellar envelope, the far side of the envelope is moving relatively toward us with respect to HL Tau at a velocity of $0.6-0.9 \mathrm{~km} \mathrm{~s}^{-1}$, while the near side of the envelope has no relative motion, or a slower relative motion at a velocity of $0.3 \mathrm{~km} \mathrm{~s}^{-1}$.

- The additional relative velocity in the protostellar envelope with respect to HL Tau is comparable to the expanding velocity of the largescale expanding shell. In addition, the additional relative velocity on the far side of the envelope, where is closer to the candidate driving source of the largescale expanding shell, is higher than that on the near side. These results suggest that the relative motion between HL Tau and its envelope could be caused by the impact of the largescale expanding motion driven by the wind or outflow from XZ Tau. Such impact likely affects the kinematics and structures of the protostellar envelope around HL Tau, and a part of the envelope likely becomes gravitational unbounded and is unlikely to be accreted onto the disk around HL Tau. If there is no other mechanism to reduce the excess angular momentum caused by the impact, our kinematical models suggest that the mass infalling rate from the envelope onto the disk in HL Tau is expected to decrease by a factor of two. Our results could demonstrate the possibility of decrease in mass infalling rates in protostellar envelopes due to outflow feedback from nearby young stellar objects.

Acknowledgements. This paper makes use of the following ALMA data: ADS/JAO.ALMA\#2015.1.00551.S. ALMA is a partnership of ESO (representing its member states), NSF (USA) and NINS (Japan), together with NRC (Canada), MOST and ASIAA (Taiwan), and KASI (Republic of Korea), in cooperation with the Republic of Chile. The Joint ALMA Observatory is operated by ESO, AUI/NRAO and NAOJ. The JCMT data were obtained under program ID M17AP086. JCMT is operated by the East Asian Observatory on behalf of The National Astronomical Observatory of Japan; Academia Sinica Institute of Astronomy and Astrophysics; the Korea Astronomy and Space Science Institute; the Operation, Maintenance and Upgrading Fund for Astronomical Telescopes and Facility Instruments, budgeted from the Ministry of Finance (MOF) of China and administrated by the Chinese Academy of Sciences (CAS), as well as the National Key R\&D Program of China (No. 2017YFA0402700). Additional funding support is provided by the Science and Technology Facilities Council of the United Kingdom and participating universities in the United Kingdom and Canada. The Starlink software Currie et al. (2014) is currently supported by the East Asian Observatory. This work is based on observations carried out under project number 143-16 with the IRAM 30m telescope. IRAM is supported by INSU/CNRS (France), MPG (Germany) and IGN (Spain). We thank all the JCMT, IRAM 30m, and ALMA staff supporting this work. This work was supported by NAOJ ALMA Scientific Research Grant Numbers 2017 04A. S.T. acknowledges a grant from the Ministry of Science and Technology (MOST) of Taiwan (MOST 102-2119- M-001-012-MY3), and JSPS KAKENHI Grant Numbers JP16H07086 and JP18K03703 in support of this work.

\section{References}

Akiyama, E., Hasegawa, Y., Hayashi, M., \& Iguchi, S. 2016, ApJ, 818, 158 ALMA Partnership (Brogan, C. L., et al.) 2015, ApJ, 808, L3
Anglada, G., López, R., Estalella, R., et al. 2007, AJ, 133, 2799

Arce, H. G., \& Sargent, A. I. 2006, ApJ, 646, 1070

Arce, H. G., Borkin, M. A., Goodman, A. A., Pineda, J. E., \& Beaumont, C. N. 2011, ApJ, 742, 105

Brittain, S. D., Rettig, T. W., Simon, T., \& Kulesa, C. 2005, ApJ, 626, 283

Cabrit, S., Guilloteau, S., Andre, P., et al. 1996, A\&A, 305, 527

Currie, M. J., Berry, D. S., Jenness, T., et al. 2014, Astronomical Data Analysis Software and Systems XXIII, ASP Conf. Ser., 485, 391

Dong, R., \& Fung, J. 2017, ApJ, 835, 146

Dong, R., Zhu, Z., \& Whitney, B. 2015, ApJ, 809, 93

Dong, R., Fung, J., \& Chiang, E. 2016, ApJ, 826, 75

Feddersen, J. R., Arce, H. G., Kong, S., et al. 2018, ApJ, 862, 121

Furlan, E., Hartmann, L., Calvet, N., et al. 2006, ApJS, 165, 568

Furlan, E., McClure, M., Calvet, N., et al. 2008, ApJS, 176, 184

Galli, P. A. B., Loinard, L., Ortiz-Léon, G. N., et al. 2018, ApJ, 859, 33

Hayashi, M., \& Pyo, T.-S. 2009, ApJ, 694, 582

Hayashi, M., Ohashi, N., \& Miyama, S. M. 1993, ApJ, 418, L71

Hirano, N., Ho, P. P. T., Liu, S.-Y., et al. 2010, ApJ, 717, 58

Jenness, T., Currie, M. J., Tilanus, R. P. J., et al. 2015, MNRAS, 453, 73

Jin, S., Li, S., Isella, A., Li, H., \& Ji, J. 2016, ApJ, 818, 76

Kanagawa, K. D., Muto, T., Tanaka, H., et al. 2015, ApJ, 806, L15

Kanagawa, K. D., Muto, T., Tanaka, H., et al. 2016, PASJ, 68, 43

Kenyon, S. J., Dobrzycka, D., \& Hartmann, L. 1994, AJ, 108, 1872

Klaassen, P. D., Mottram, J. C., Maud, L. T., \& Juhasz, A. 2016, MNRAS, 460, 627

Krist, J. E., Stapelfeldt, K. R., Burrows, C. J., et al. 1999, ApJ, 515, L35

Krist, J. E., Stapelfeldt, K. R., Hester, J. J., et al. 2008, AJ, 136, 1980

Lee, C.-F., Mundy, L. G., Reipurth, B., Ostriker, E. C., \& Stone, J. M. 2000, ApJ, 542,925

Li, Z.-Y., Banerjee, R., Pudritz, R. E., et al. 2014, Protostars and Planets VI (Tucson, AZ: University of Arizona Press), 173

Li, H., Li, D., Qian, L., et al. 2015, ApJS, 219, 20

Loinard, L. 2013, Advancing the Physics of Cosmic Distances (Cambridge: Cambridge University Press), 289, 36

Lumbreras, A. M., \& Zapata, L. A. 2014, AJ, 147, 72

Machida, M. N., \& Matsumoto, T. 2012, MNRAS, 421, 588

Machida, M. N., Inutsuka, S.-i., \& Matsumoto, T. 2010, ApJ, 724, 1006

Machida, M. N., Inutsuka, S.-I., \& Matsumoto, T. 2011, PASJ, 63, 555

Mangum, J. G., \& Shirley, Y. L. 2015, PASP, 127, 266

McMullin, J. P., Waters, B., Schiebel, D., et al. 2007, Astronomical Data Analysis Software and Systems XVI, eds. R. A. Shaw, F. Hill, \& D. J. Bell (San Francisco, CA: ASP), 127

Men'shchikov, A. B., Henning, T., \& Fischer, O. 1999, ApJ, 519, 257

Monin, J.-L., Pudritz, R. E., \& Lazareff, B. 1996, A\&A, 305, 572

Motte, F., \& André, P. 2001, A\&A, 365, 440

Movsessian, T. A., Magakian, T. Y., \& Moiseev, A. V. 2012, A\&A, 541, A16

Offner, S. S. R., \& Arce, H. G. 2015, ApJ, 811, 146

Pineda, J. L., Goldsmith, P. F., Chapman, N., et al. 2010, ApJ, 721, 686

Smith, R. L., Pontoppidan, K. M., Young, E. D., \& Morris, M. R. 2015, ApJ, 813, 120

Shu, F. H. 1977, ApJ, 214, 488

Shu, F. H., Ruden, S. P., Lada, C. J., \& Lizano, S. 1991, ApJ, 370, L31

Shu, F. H., Najita, J. R., Shang, H., \& Li, Z.-Y. 2000, Protostars and Planets IV (Tucson, AZ: University of Arizona Press), 789

Takakuwa, S., Ohashi, N., \& Hirano, N. 2003, ApJ, 590, 932

Takakuwa, S., Kiyokane, K., Saigo, K., \& Saito, M. 2015, ApJ, 814, 160

Takami, M., Beck, T. L., Pyo, T.-S., McGregor, P., \& Davis, C. 2007, ApJ, 670, L33

Testi, L., Skemer, A., Henning, T., et al. 2015, ApJ, 812, L38

Ulrich, R. K. 1976, ApJ, 210, 377

Visser, R., van Dishoeck, E. F., Doty, S. D., \& Dullemond, C. P. 2009, A\&A, 495, 881

Visser, R., Doty, S. D., \& van Dishoeck, E. F. 2011, A\&A, 534, A132

Vorobyov, E. I. 2010, ApJ, 723, 1294

Vorobyov, E. I. 2011, ApJ, 729, 146

Vorobyov, E. I. 2016, A\&A, 590, A115

Welch, W. J., Hartmann, L., Helfer, T., \& Briceño, C. 2000, ApJ, 540, 362

Wilson, T. L., \& Rood, R. 1994, ARA\&A, 32, 191

Wu, C.-J., Hirano, N., Takakuwa, S., Yen, H.-W., \& Aso, Y. 2018, ApJ, 869, 59

Yen, H.-W., Liu, H. B., Gu, P.-G., et al. 2016, ApJ, 820, L25

Yen, H.-W., Takakuwa, S., Chu, Y.-H., et al. 2017, A\&A, 608, A134

Zapata, L. A., Galván-Madrid, R., Carrasco-González, C., et al. 2015, ApJ, 811, L4

Zhu, Z., Hartmann, L., Nelson, R. P., \& Gammie, C. F. 2012, ApJ, 746, 110 


\section{Appendix A: Initial particle distributions in our kinematical models}

Figure A.1 presents the distributions of the test particles having different line-of-sight velocities projected on the plane of the sky in the kinematical model with $V_{\text {rel }}$ of $0.6 \mathrm{~km} \mathrm{~s}^{-1}$ starting with an axisymmetric initial particle distribution after evolution of $10^{4} \mathrm{yr}$ in comparison with the observed velocity channel maps. At $V_{\mathrm{LSR}}$ of $4.7-5.4 \mathrm{~km} \mathrm{~s}^{-1}$, the particle distributions overlap with the blueshifted arc-like structure in the observations, suggesting that including the relative motion between HL Tau and its protostellar envelope can explain the observed high-velocity in the blueshifted arc-like structure. Nevertheless, the particle distributions in the kinematical model do not show similar morphologies to the observed arclike structures. Those particles that do not overlap with the observed intensity distributions above the $3 \sigma$ level are removed from the initial particle distribution, resulting in the model envelopes with asymmetric initial density distributions shown in Fig. 11.

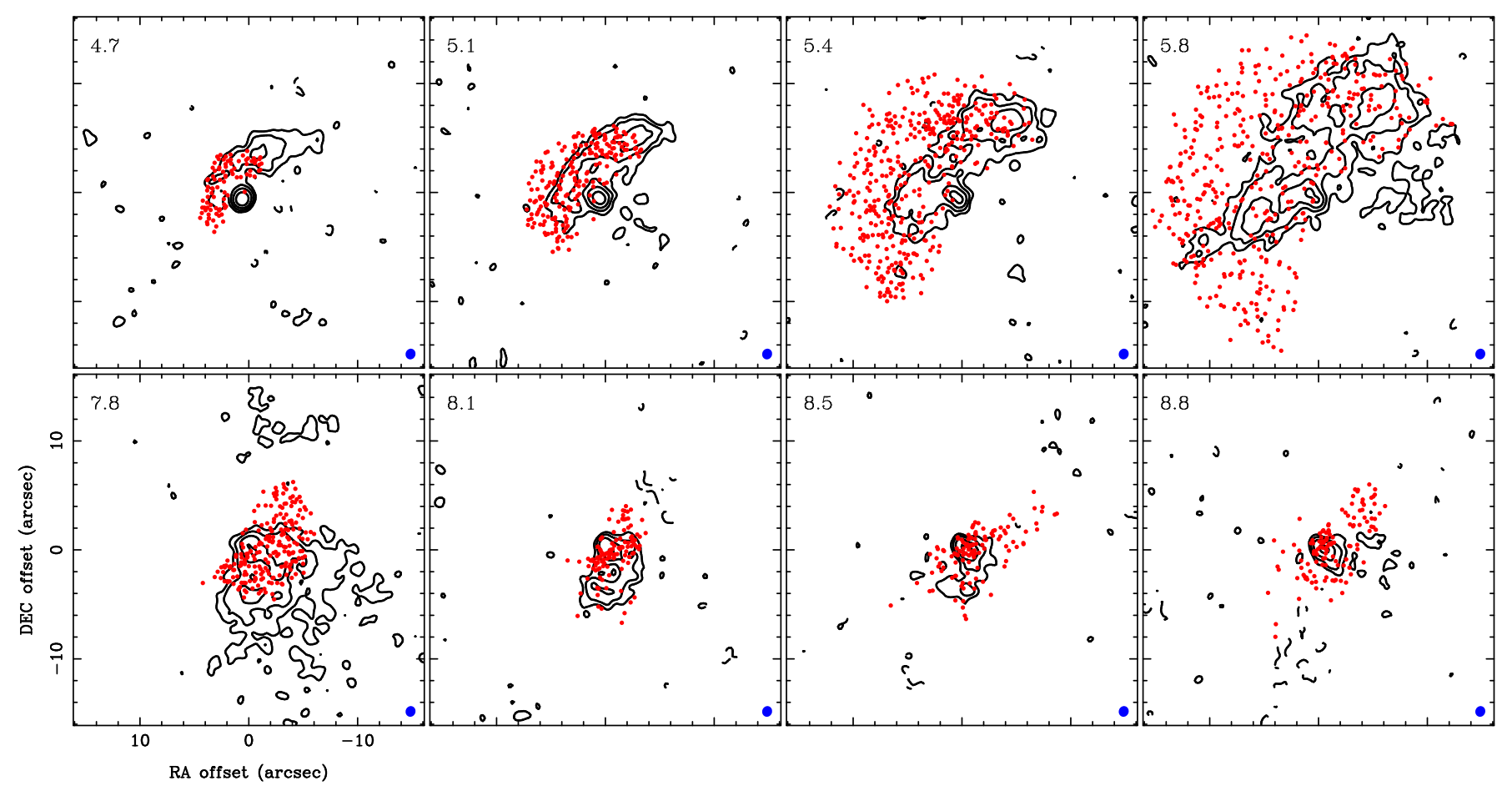

Fig. A.1. Observed velocity channel maps of the $\mathrm{C}^{18} \mathrm{O}$ emission (contour) overlaid with the particle distributions (red dots) at the same line-of-sight velocities from our kinematical model with $V_{\text {rel }}$ of $0.6 \mathrm{~km} \mathrm{~s}^{-1}$ after an evolutionary time of $10^{4} \mathrm{yr}$. Contour levels are from $3 \sigma$ and increase in steps of a factor of two, where $1 \sigma$ is $5.5 \mathrm{mJy} \mathrm{Beam}^{-1}$. The central velocity of each channel is labeled at the upper left corner in each panel in units of $\mathrm{km} \mathrm{s}^{-1}$. Blue filled ellipses show the size of the synthesized beam. 


\section{Appendix B: Synthetic images of different envelope models}

For comparison, Figs. B.1 and B.2 present the synthetic velocity channel maps generated from the kinematical models with $V_{\text {vel }}$ of 0.3 and $0.9 \mathrm{~km} \mathrm{~s}^{-1}$.

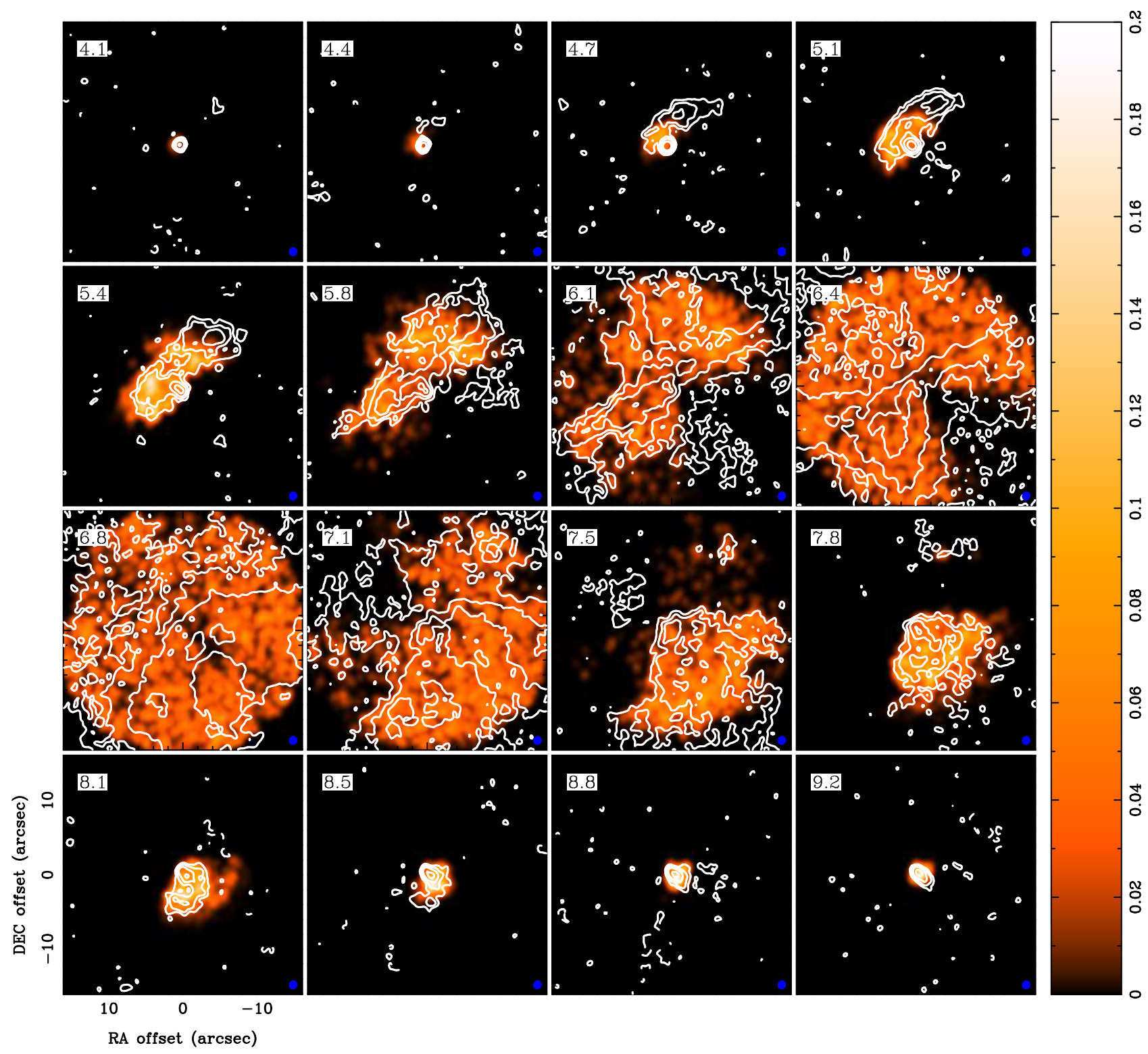

Fig. B.1. Same as Fig. 12 but with $V_{\text {rel }}$ of $0.3 \mathrm{~km} \mathrm{~s}^{-1}$. 


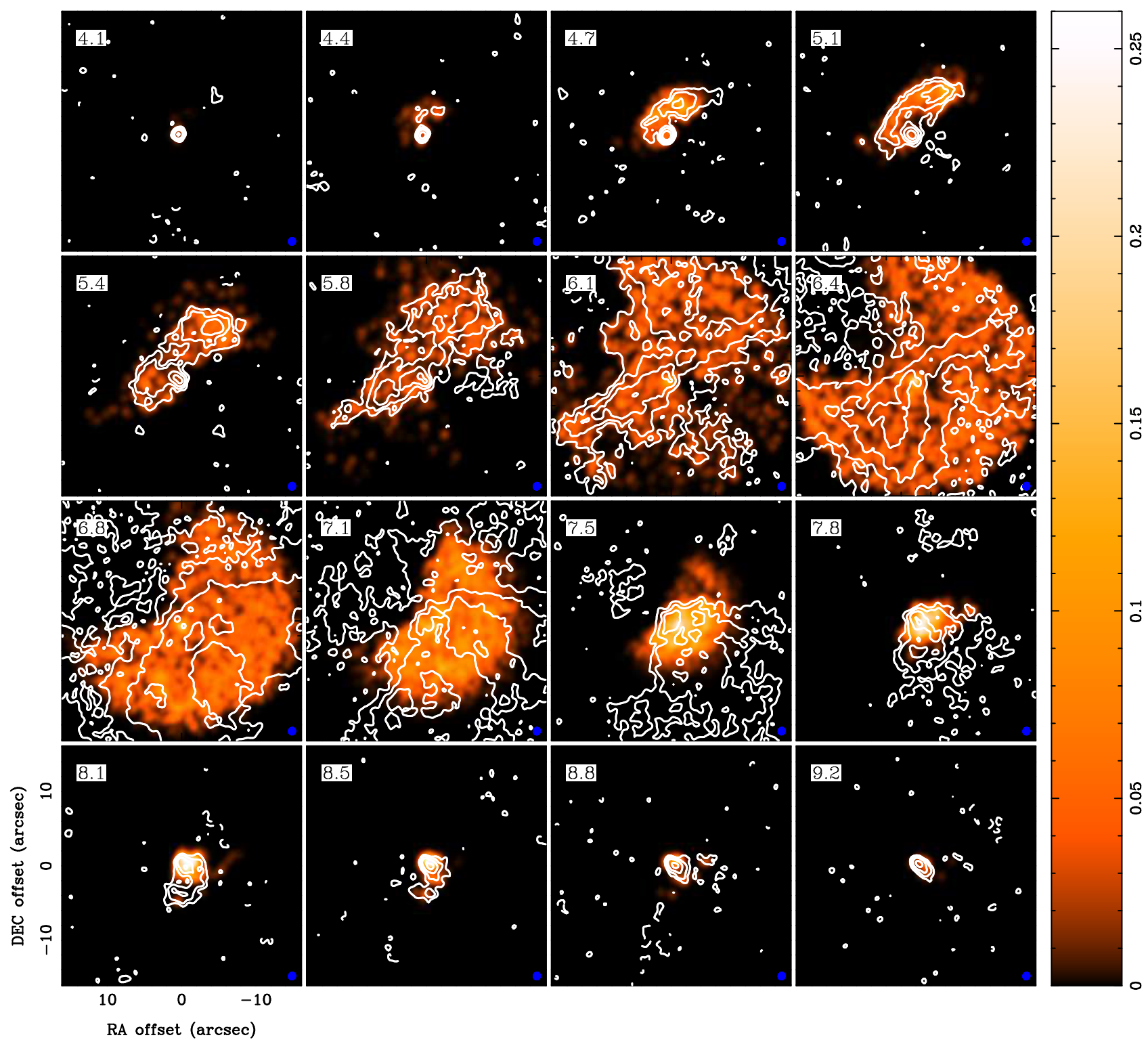

Fig. B.2. Same as Fig. 12 but with $V_{\text {rel }}$ of $0.9 \mathrm{~km} \mathrm{~s}^{-1}$. 